\title{
Local Computed Tomography Using a THz Quantum Cascade Laser
}

\author{
Xiaoxia Yin, Student Member, IEEE, Brian Wai-Him Ng, Member, IEEE, J. Axel Zeitler, Kieu Lien Nguyen, \\ Lynn F. Gladden, and Derek Abbott, Fellow, IEEE
}

\begin{abstract}
This paper investigates local reconstruction techniques for extracting region-of-interest (ROI) from a 3-D terahertz imaging setup using a quantum cascade laser (QCL). The advantage of local reconstruction is the reduction in the required measurement time. Difficulties with limited projection angles and image noise make the development of accurate reconstruction algorithms particularly challenging. In this paper, both wavelet-based and traditional filtered back projection (FBP) techniques are investigated. Segmentation algorithms are applied to reconstructed images with low contrast and the resultant segments are compared with a known ground truth to explore the ability of a QCL to image target objects with complex contours. In our experiments, a polystyrene object with a hole drilled inside is used as the imaging target. The region of interest is adjusted through changing the size of the exposure regions. It is found that 3-D local reconstruction of the interior hole suffers from shape distortion, since scattering caused by the target's exterior contours introduces errors in the measured optical parameters of the object. It is found that wavelet-based local computed tomography for terahertz image reconstruction results in lower misclassification of pixels than traditional FBP algorithms.
\end{abstract}

Index Terms-Computed tomography, discrete wavelet transforms (DWT), filtered back projection, fuzzy c-means threshold, quantum cascade laser (QCL), terahertz.

\section{INTRODUCTION}

Q UANTUM CASCADE LASERS (QCLs) are semiconductor injection lasers, based on quantum semiconductor structures that are grown by molecular beam epitaxy and designed by band structure engineering [1], [2]. QCLs, in principle, are realized via intersubband transitions in a multiplequantum-well (MQW) heterostructure [3], [4]. In contrast to conventional diode lasers, QCLs only involve electron transitions that occur between the conduction bands (intersubband)

Manuscript received November 09, 2009; revised February 05, 2010. Date of publication June 07, 2010; date of current version September 15, 2010. Part support from the Australian Research Council Nanotechnology Network funded the collaborative visit to the University of Cambridge. This work was supported in part by the Australian Research Councils (ARC) Discovery Project funding scheme under Project No. DP0988064. The associate editor coordinating the review of this paper and approving it for publication was Prof. Francisco Arregui.

X. Yin, B. W.-H. Ng, and D. Abbott are with the Centre for Biomedical Engineering and the School of Electrical and Electronic Engineering, University of Adelaide, SA 5005, Australia (e-mail: xxyin@eleceng.adelaide.edu.au; bwng@eleceng.adelaide.edu.au; dabbott@eleceng.adelaide.edu.au).

J. A. Zeitler K. L. Nguyen, and L. F. Gladden are with the Department of Chemical Engineering, University of Cambridge, Cambridge CB2 3RA, U.K. (e-mail: jaz22@cam.ac.uk; lfg1@cam.ac.uk).

is with the Department of Chemical Engineering, University of Cambridge, Pembroke Street, Cambridge CB2 3RA, U.K.

Color versions of one or more of the figures in this paper are available online at http://ieeexplore.ieee.org.

Digital Object Identifier 10.1109/JSEN.2010.2045754 rather than between the conduction and valence bands [5]. As a result, the emission wavelength can be controlled by the thickness of the MQW, and the intrinsic high-power capabilities of the lasers make possible the cascading process, in which each electron generates several tens of photons in superlattice structures [6]. In addition, intersubband transitions are characterized through ultrafast carrier dynamics and band-structure engineering is available to successfully control the electron flow and thus increase population inversion, which ultimately controls the laser threshold [3], [7].

Terahertz radiation (T-ray or $\mathrm{THz}$ ) spans the frequency range from $0.1 \mathrm{THz}$ to $10 \mathrm{THz}$ in the electromagnetic spectrum [8]. The high power levels obtainable from Quantum Cascade Lasers (QCL) give them an advantage for biomedical imaging and security detection applications [9]. Terahertz imaging is performed using either terahertz pulsed imaging (TPI) or continuous wave $(\mathrm{CW})$ terahertz modes. Time-domain TPI has the advantage of providing a broad frequency spectrum, and resolving the arrival of the photons as a function of time at different locations around the specimen boundary, but this has an obvious drawback in the inability to generate a narrow linewidth spectrum. By contrast with $\mathrm{THz}$ pulsed imaging, QCLs afford high power sources of continuous wave (CW) radiation to achieve deeper penetration of samples, which addresses one of four principal challenges for terahertz tomography [10]. The $\mathrm{CW}$ terahertz imaging method uses $\mathrm{THz}$ radiation of constant intensity and offers improved signal-to-noise performance but with the difficulty of resolving material absorption from scattering [11]. We recognize that there are still challenges for QCLs to reach higher operating temperatures and higher signal-to-noise ratio (SNR), nevertheless, several tens of milliwatt peak terahertz power [12] and a few tens of $\mathrm{dB}$ of SNR can already be very useful for biomedical imaging and other applications. This paper aims to examine the optical characteristics regarding a $\mathrm{CW} \mathrm{THz} \mathrm{QCL}$, which illuminates an object with heterogeneous layers and complex contours in a volume space.

Terahertz QCLs were first reported only recently [5]. This is due to an insuperable barrier that exists in the phonon reststrahlen band, which causes difficulty in the generation of lower terahertz frequencies. Moreover, the development of a suitable waveguide [13] is necessary. It aims to confine long wavelength T-rays to an epilayer with low absorption losses by applying molecular beam epitaxy technology [6]. Current new design concepts have led to continuous-wave operation and high output powers [14]. Recently, terahertz QCLs have made rapid progress, from depending on chirped superlattices [15] and the surface plasmon concept [16] employed for a large optical confinement without imposing high propagation losses, to recently, bound-to-continuum transitions and extraction of 
carriers, via resonant phonon scattering [17]. Recent progress concerning terahertz single-mode operation and frequency extension has been reported [6].

T-rays are non-ionizing, unlike $\mathrm{X}$-rays, while T-ray imaging allows us to image objects through dry, non-metallic layers of packing. Currently, THz techniques also show some promise in competition against MRI. An example is that $\mathrm{THz}$ opens up the possibility of endoscopic detection of cancer in the internal organs [18], [19], which is not possible with MRI. It should be emphasized that unlike MRI and X-ray imaging, which realize parallel scanning using multiple point detection, the fundamental scheme of terahertz scanning uses point to point detection. In order to obtain an image, it is necessary to perform many individual scans serially. Thus, it is an issue of practical significance to reduce the measurement time of each scan. Often, we are only interested in reconstructing a subregion of an object, or what is commonly referred to as the region of interest (ROI). Imaging can then be sped up by simply scanning the ROI instead of the entire object. However, this simple approach is known to lead to artifacts in reconstruction, due to the global nature of the standard reconstruction technique. The main goal of this paper is to present and compare a wavelet-based reconstruction algorithm with the standard techniques for computing localized reconstructions and to show how this algorithm can be used to rapidly reconstruct the ROI with a reduction in the measurements of terahertz responses. Though to some extent, the reconstruction results are noisy, the current reconstruction algorithm yet clearly paves the way to more advanced algorithms for local recovery of T-ray images, especially based on a T-ray QCL. The advanced algorithms proposed could be based on basin inversion in achieving local reconstruction with reduced projection angles and $a$ priori, to more recent advanced image processing techniques, such as compressive subsampling, which can be used to recover a T-ray image with sparse T-ray measurements. The current wavelet reconstruction algorithm will also have an impact on the exploration of traditional T-ray hardware design that, to some extent, has significant limitations, especially in achieving a full collection of desirable discrete-time samples.

This paper is organized as follows. Section II briefly introduces THz QCL imaging and overviews the methodology used for computed tomography and wavelet-based local computed tomography. Section III systematically implements the methodology on a target specimen, a polystyrene clown's head with a hole inside. The local reconstruction techniques are analyzed and validated via segment strategies. Reconstruction results related to different algorithms are discussed in Section IV, which consist of error calculations in the reconstructed slope and the number of pixels in reconstructed images, compared to the ground truth. Meanwhile, the values of radii with respect to the Region of Exposure (ROE) and ROI at different heights are also discussed in this section in regard to local reconstruction using these algorithms.

\section{Methodology}

\section{A. An Overview of Computed Tomography}

Computed tomography produces sectional images of an object. It is based on the assumption that the image to be reconstructed belongs to an identifiable ensemble of similar images
[20]. The filtered back projection algorithm is often used in the calculation of the inverse Radon transform [21]-[23], which reconstructs a target object from a collection of projections. Typically, the quantities obtained are the object's frequency dependent optical properties, such as the absorption coefficients. Normally, a filtered back projection algorithm begins with a collection of sinograms obtained from projection measurements. A sinogram is a collection of points in a projection space. It is generated using Radon transform data, and is simply a collection of the projections at all the projection angles. A 1-D projection operation at each projection angle is a linear integral of the image intensity along the projection offset.

\section{B. T-Ray Quantum Cascade Laser Setup}

A schematic diagram of the current QCL imaging apparatus is illustrated in Fig. 1(a). The pulse generator supplies pulses at a frequency of $80 \mathrm{kHz}$ to the QCL. The output signal from the pulse generator is usually gated with a $15 \mathrm{~Hz}, 50 \%$ duty cycle, slow modulation by an optical chopper to match the detector (the Golay cell) response time, and to offer a reference frequency to the lock-in amplifier (LIA). The LIA is used to digitize signals and to significantly improve the signal-to-noise ratio by setting a time constant, over which the input signal is integrated for each data point. The optimal time constant is set to $50 \mathrm{~ms}$ and a threshold current density is set to $112 \mathrm{~A} / \mathrm{cm}^{2}$.

The terahertz QCL used is a GaAs-AlGaAs bound-to-continuum superlattice design, emitting at $2.9 \mathrm{THz}(103 \mu \mathrm{m})$, and grown by molecular beam epitaxy [12]. This device typically operates up to $95 \mathrm{~K}$ in pulse mode and delivering $70 \mathrm{~mW}$ peak power per facet. The QCL is mounted on the cold finger of a continuous-flow helium-cooled cryostat maintaining a heat-sink temperature of $4.2 \mathrm{~K}$. The emission is collected with a 2 " $\mathrm{f} / 1$ off-axis parabolic mirror, then focused by a 2 " $\mathrm{f} / 6.43$ parabolic mirror onto the sample. The sample is mounted on a rotational stage, which is itself mounted on an $x y z$ translational stage (two directions $x$ and $z$ for raster scanning the target and a third direction $y$ for the optical delay line). This current terahertz imaging setup achieves point to point detection. The linearly moving stage enables parallel scanning along the $x$ axis with a linear velocity of $20 \mathrm{~mm} / \mathrm{s}$ and a rotating stage, labelled $\theta$, allows projections of an object to be taken at a number of projection angles with a rotary velocity of 4 degree/s. The $y$ axis is perpendicular to the paper. The $z$ axis motion allows the sample to move vertically in order to obtain cross-sectional images at various heights. The transmitted beams are detected by a Golay cell. The power incident on the sample, including the effects of the transmission of the cryostat window, is typically $\sim 35 \mathrm{~mW}$ (peak). The signal-to-noise ratio in the absence of a sample in the beam is 20 $\mathrm{dB}$ [24]. The photograph of a part of the terahertz QCL imaging system is shown in Fig. 1(b).

\section{2-D Wavelet Based CT Reconstruction}

1) 2-D Discrete Wavelet Transform: Wavelet transforms (WTs) play an important role in many image processing tasks. Fundamentally, wavelet decomposition corresponds to a multiresolution analysis of a signal. This has the advantage of much improved joint time-frequency localization over Fourier-based techniques. In most applications, it is nearly 


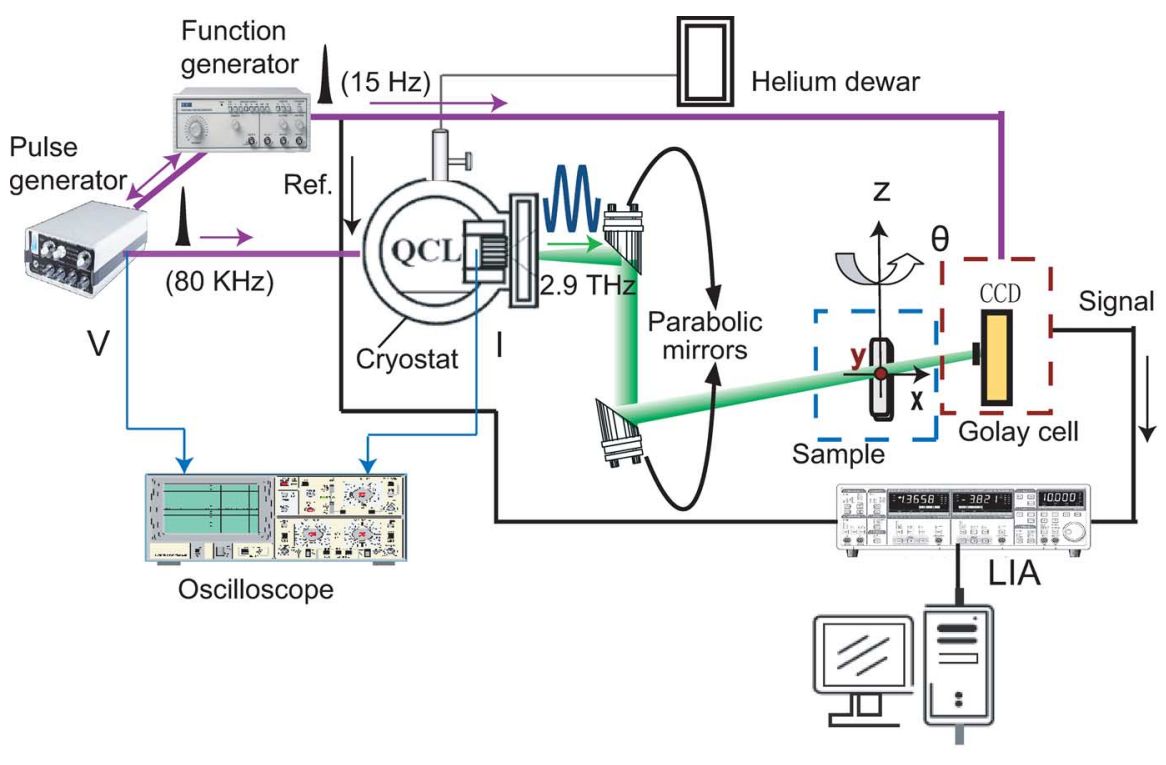

(a)

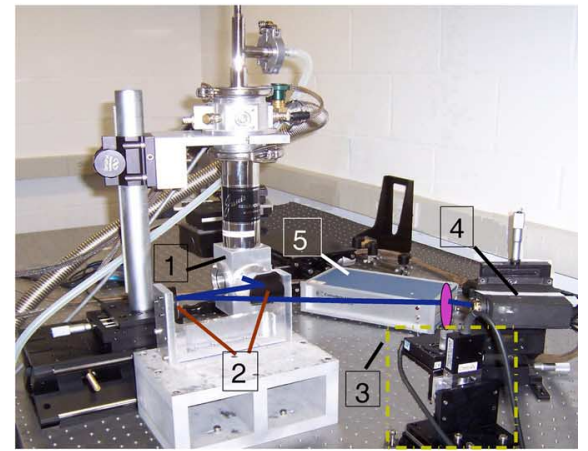

(b)

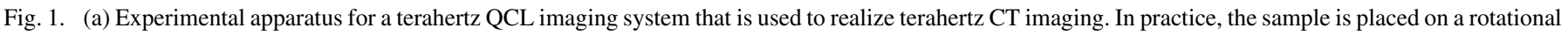

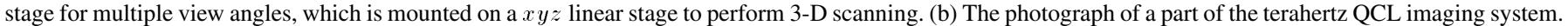

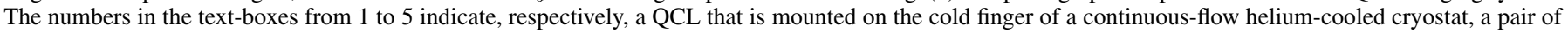
parabolic mirrors, the rotational stage mounted on an $x y z$ translational stage, a Golay cell detector, and a detector controller.

always implemented using digital filters and downsamplers. In two dimensions, the discrete version of a wavelet transform can be realized by a 2-D scaling function, and three 2-D wavelets, which are calculated by taking the 1-D wavelet transform along the rows of an image and the resulting columns [25]. Then, the convolved images are downsampled to yield low-resolution approximate and detail coefficients. This process can be repeated to yield successively lower resolution images. In 1-D, the basis consists of translation of a function $\phi(x)$, called the scaling function, together with translations and dyadic scalings of a function $\psi(x)$, called a wavelet. In 2-D, a similar basis using the scaling function $\phi(x, y)=\phi(x) \phi(y)$ together with dyadic scalings and integer translations of the three wavelets $\psi_{H}(x, y)=\phi(x) \psi(y), \psi_{V}(x, y)=\psi(x) \phi(y)$, $\psi_{D}=\psi(x) \psi(y)$, where the subscripts indicate that these wavelets are to represent edges that are oriented horizontally, vertically, and diagonally, respectively [26].

2) 2-D Wavelet Reconstruction: This section briefly describes our algorithm, which is applied to obtain the wavelet coefficients of a function on $\mathbf{R}^{2}$ space, based on Radon transform data. This method enables reduced computation load compared to the wavelet coefficients obtained by taking wavelet transforms on the reconstructed image. Moreover, the wavelet coefficients can be directly computed from the sinograms, which then allow the target to be reconstructed to yield local computed tomography [27]. The main algorithm and derivation of computing 2-D DWTs from the projection data were introduced in [27]. Quite simply, they are realized via performing separate wavelet transforms on 1-D projection data, which amounts to a conventional inversion of the Radon transform with the ramp filter replaced by the wavelet ramp filter(s). This processing aims to expand each of the projections in a wavelet transform in order to localize the Hilbert transform, and therefore the filtered back projection algorithms [28].
In this paper, only one 2-D wavelet transform step is used. This is because the single level decomposition of scaling and wavelet ramp filters allows clear reconstruction of an image in the ROI and it avoids further computational complexity due to increased levels of WTs [27], [29]. The 2-D inversion of the traditional wavelet transform is conducted on the back projection of reconstructed approximate and detail sinograms, after the decomposition procedure is performed.

3) Local Reconstruction Using Wavelets: A significant characteristic of most wavelet transforms is the existence of a number of vanishing moments. Hilbert transforms of functions with many vanishing moments have been shown to decay very rapidly at infinity [29]. In other words, a wavelet function with compact support allows a local basis to maintain its localized features after Hilbert transformation [29]. Therefore, the wavelet and scaling coefficients for a wavelet basis can be calculated after applying the projections passing through the region of interest (ROI) plus a margin for the support of the wavelet and scaling ramp filters. We call this region (ROI plus a margin), the region of exposure (ROE). These reconstructed coefficients, in this experiment, are then directly applied to the inverse wavelet transforms for terahertz image reconstruction.

\section{IMPLEMENTATION}

\section{A. Experimental Considerations}

In this paper, one set of terahertz QCL data is considered for Local Computed Tomography (LCT): a nested structure of a polystyrene clown's head with a hole inside, see the target photo in Fig. 2(a) and (b). The target sample is imaged in 12 slices, from bottom to top at 12 different heights, $5 \mathrm{~mm}$ apart. The diameter of the hole is $10.1 \pm 0.2 \mathrm{~mm}$, measured directly from the target. For the current local reconstruction, only the second to the fifth image slices are considered with the hole going through 


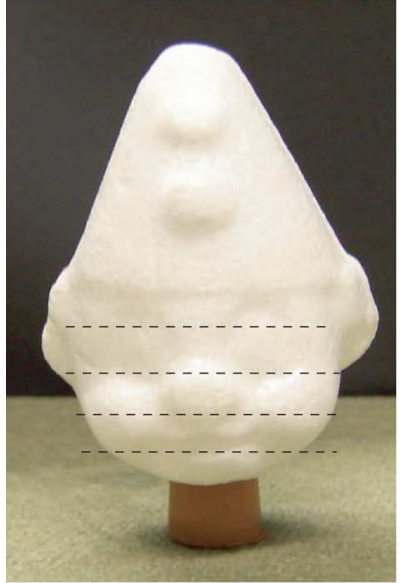

(a)

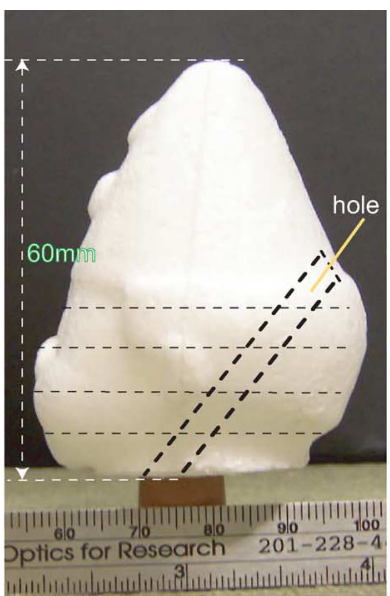

(b)

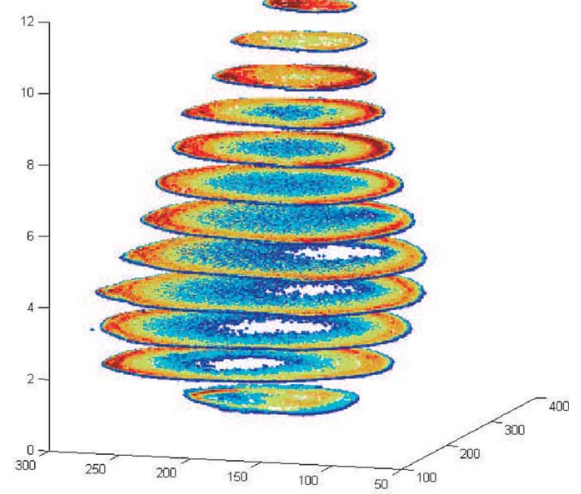

(c)

Fig. 2. (a) The photograph of a nested structure of a polystyrene clown's head with hole inside. (b) The photograph of the side face of the polystyrene clown's head. (c) The reconstructed slices are stacked to recover the nested structure of the target object with a hole inside. The lines in (a) and (b) indicate the current four sliced heights for LCT in the hole area of interest.

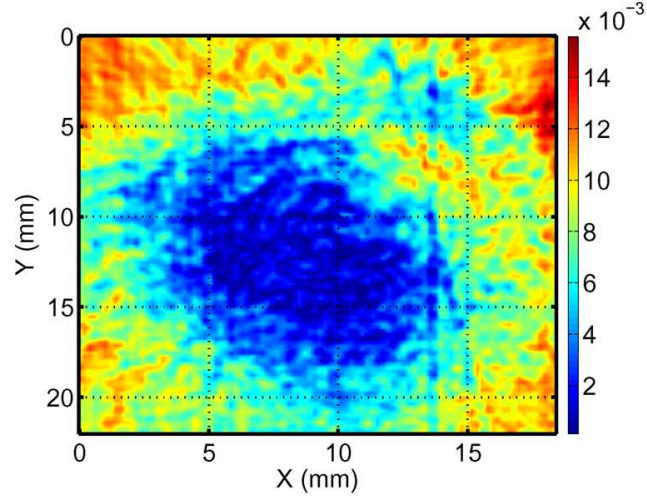

(a)

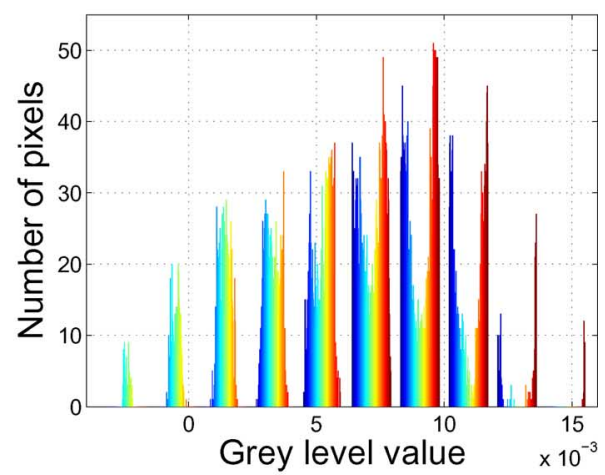

(b)

Fig. 3. (a) Illustration of a typical local reconstruction of a region of the target sample containing a single hole. (b) The intensity histogram of the relative image of (a) is depicted.

at a tilted angle of $43^{\circ}$. The center of the hole at the first slice is also centered at the bottom cross-section. The first three target layers (from the second to the fourth image slice), labelled Slices 1 to 3 , are imaged using 289 projections at each of 18 projection angles covering a $180^{\circ}$ projection area to produce a $250 \times 250$ pixel image (edge truncated), while the fifth slice (Slice 4 ) is imaged using 268 projections at the same 18 projection angles. Fig. 2(c) shows the reconstructed slices, which are stacked to recover the nested structure of the target object, where the lines indicate that four sliced heights which are used for the exploration of the current local reconstruction of the hole sample.

\section{B. Error Analysis}

In order to test the performance of the local imaging system, only binary grey-level images are used via segmentation techniques, after back projection processes, including a truncated version of global CT via FBP, LCT via wavelet transforms, and LCT via FBP, are carried out in the region of interest. The pixel count difference is calculated between the ground truth and each of the resultant segments at four different measurement heights. The error ratio is regarded as the difference divided by the total number of pixels of the binary grey-level image from the target sample. In turn, for further validation of our segmentation scheme, slopes computed from the centroids of the segments at two different slices are used to compute the error of our reconstruction, by comparing with the true slope arising from the linear 3-D (hole) structure.

1) Characteristics of a Hole Image: A typical local reconstruction, containing a single hole, is shown in Fig. 6(a). The intensity histogram of the relative image is depicted in Fig. 6(b).

The typical local reconstruction concerns a single hole through the sample. There are several notable characteristics of this image, which are common to local reconstructions of the hole embedded in a 3-D target at the different slices by different image reconstruction algorithms. First, the contrast of intensities among the hole, the polystyrene clown's head slice (object) and background is low. This is evident from the intensity histogram in Fig. 3(b). However, the average intensity of the object and background is often roughly uniform, with the hole embedded in the target cross-section shown as a slightly darker region. Second, it is observed that the hole embedded in the target physically distorts the measured optical parameters 
of the object, depending on the slice shape and hole distance from the detector at each different measurement height. The varying exterior boundaries of the different slices result in varying degrees of optical distortion due to light scattering. In addition, a longer path length from the hole center position at each slice to the laser results in larger absorption. The 3-D local reconstruction of the hole target takes on a number of different shapes in the different slices. As a result, the boundary of the hole is potentially difficult to define. Due to variations in intensity, both within the hole position and in the background, portions of the hole appear to blend directly into the background, without creating a distinct boundary.

2) Segmentation Strategy: The aim of segmentation is to separate an image into different meaningful regions, by identifying the regions in an image with common properties and distinguishing dissimilar regions [30]. A popular segmentation scheme is image thresholding [25], [31], which can be regarded as a form of per-pixel classification. Gray-level intensity is used as a feature value to characterize each pixel of an image. The calculated threshold is compared with the feature value in order to map the pixel onto one of two groups, object and background regions.

In this paper, two threshold levels are used to segment the entire image, as the recovered image, e.g., Fig. 3(a), shows a gradual change in intensity from the three regions: the hole, the polystyrene clown's head cross-section, and the background. Since separation of different clusters is not well-defined, with dependence on the local grey value distribution, a fuzzy c-means technique is applied in this paper, associated with selected a set of feature vectors that are strictly local in nature, that is, they depend on the 8-neighborhood of the pixel concerned. The fuzzy c-means (FCM) method is advantageous for automatically computing two or more optimal segmentation thresholds, and for achieving the separation of underlying distributions. A fuzzy c-means threshold provides an iterative measurement to minimize the classification error allowing each pixel to be the member of all the possible classes with varying membership.

Because of noise, as stated below, additional fuzziness is introduced in the pixel value, while selecting it to be a member of one of several classes. According to [32], [33], the FCM algorithm assigns pixels to each category by using fuzzy memberships. Let $X=\left(x_{1}, x_{2}, \ldots, x_{N}\right)$ denote an image with $N$ pixels to be partitioned into $c$ clusters.

The algorithm minimizes the cost function iteratively to find the best location for each of the clusters. The cost function satisfies a least-squared error criterion

$$
J=\sum_{j=1}^{N} \sum_{i=1}^{c} u_{i, j}^{m}\left\|x_{j}-v_{i}\right\|^{2}
$$

where $u_{i, j}$ represents the membership of pixel $x_{j}$ in the $i^{t h}$ cluster; $v_{i}$, whose number of components depends on the number of feature vectors (make reference to [32]), is the cluster center of $i^{\text {th }}$ class of fuzzy c-partitions, also called seed point for the particular class. The results of the calculated error or cost function are updated in every iteration according to $u_{i, j}$ and the distance $\left\|x_{j}-v_{i}\right\|$. This continues till the cluster centers become stable without significant difference between cluster centers in two consecutive iterations. The weighting exponent $m$ is a constant with $m$ equal to or bigger than 1 , which controls the fuzziness of the resulting partition. The exponent of $u_{i, j}$ equal to 2 and maximum number of iterations equal to 100 are studied. The cost function is minimized when pixels close to the centroid of their clusters and are assigned high membership values, and vice versa. The membership function represents the probability that a pixel belongs to a specific cluster. In the FCM algorithm, the probability is determined by the distance between the pixel and each individual cluster center in the feature domain. The membership functions and cluster centers are updated according to the follows:

$$
u_{i, j}=\frac{1}{\sum_{k=1}^{c}\left(\frac{\left\|x_{j}-v_{i}\right\|}{\left\|x_{j}-v_{k}\right\|}\right)^{2 /(m-1)}}
$$

and

$$
v_{i}=\frac{\sum_{j=1}^{N} u_{i, j}^{m} x_{j}}{\sum_{j=1}^{N} u_{i, j}^{m}} .
$$

Equations (2) and (3) present iterative procedures of FCM. The iterative procedures start from an arbitrary assigned initial cluster center $v_{i}$, which leads to a solution that FCM converges to. The solution represents the local minimum or a saddle point of the cost function. Convergence varies with the changes in the membership function or the cluster center at two successive iteration steps. Fig. 4(a) and (b) are the resultant segments between the hole region and the region of object (including background), as well as the segments between object region and background, using two different calculated thresholds, separately. In this paper, since we only consider a local segment of the hole region, so we focus on thresholding just for the hole, which is followed by postprocessing to clean up the segmented region. For convenience, in the following, we call the region of object adjoined to background as background.

Generally, thresholding an image can incur two kinds of noise: background pixels misclassified as a hole pixel, which produce small disjoint hole components in addition to the hole region, and hole pixels misclassified as the background, which produce gaps in the hole region of interest, see Fig. 4(b). Misclassification of either target sample of interest or background pixels near the hole boundary can also produce an extremely coarse boundary. In order to obtain the boundary representation of the hole region, region-based segmentation operations and morphological operations [25] are combined to repair the gaps in the hole region of interest and improve the smoothing of boundaries. This approach aims to apply region growing on a typical local reconstruction, which is represented as follows. Eight neighbor pixels are used as seed pixels. There are four starting points from four sets of seed pixels, which are applied for segmentation. Those starting points are positioned at the central point of the LCT in ROI or the point around the center. The four sets of seed pixels move from pixel to pixel along four quadrants of the coordinates, separately, starting from the four starting points. Predefined criteria are selected depending on the broken characters that are shown in each 


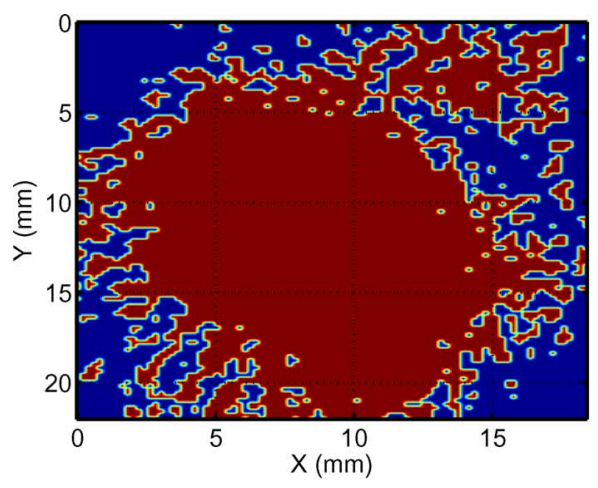

(a)

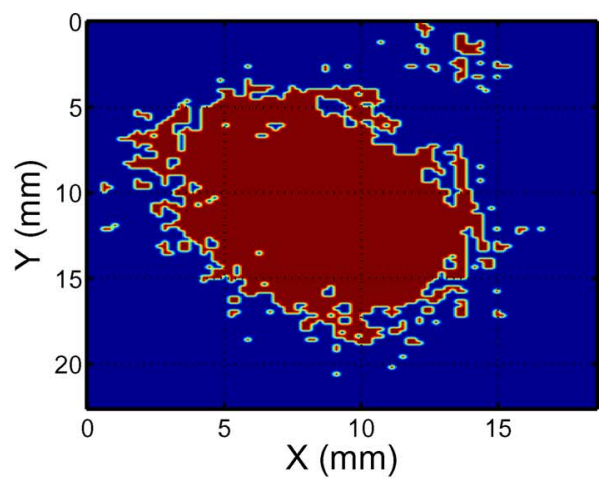

(b)

Fig. 4. Illustration of the result of applying two calculated thresholds via the fuzzy c-means thresholding method on the image of Fig. 3(a). (a) The image between the object region and background, achieved via using one of the two calculated thresholds. (b) The segmented image achieved via using the other threshold.

reconstruction. The maximum length of the breaks in the eight neighboring pixels is set to be two to four pixels. During the processing, we assign 1 to the pixel satisfying the selection criteria, otherwise we set the pixel to zero. This region growing is conducted repeatedly till a single connected hole region of interest appears without gaps.

In some cases, artifacts can be mistaken for the hole region of interest. After region growing is used on regions obtained by thresholding the local reconstruction, a simple criterion is adopted to examine the length feature of the segmented hole region of interest, to ensure that the correct hole target region is selected. In this way, large artifacts can be eliminated, and small artifacts can be removed by region growing.

To calculate the error of segmentation-based reconstruction, we compare each of the resultant segments with the ground truth. The error ratio is computed as the number of different pixels between the resultant segment and ground truth divided by the number of pixels of the segment from the ground truth. The ground truth is computed from the physical measurements of the target and using the planar projection of the tubular hole structure-ellipses with semimajor axes of $13.2 \pm 0.6 \mathrm{~mm}$ and semiminor axes of $10 \pm 0.8 \mathrm{~mm}$ along with the rotation angle of $8 \pm 2^{\circ}$.

Fig. 7 illustrated the segmentation processes regarding the planar projection of the tubular hole structure. It should be noted that in the error ratio calculation, what we are concerned with is the difference of the number of pixels between ground truth and reconstructed segments at each layer. For uniformity, we try to match the reconstructed segments with ground truth. That is, the position of the centroid of the ground truth is always moved to guarantee an overlapping with the centroid of each reconstructed segment at every segment height.

\section{RECONSTRUCTION RESULTS}

Four slices are analyzed for this target sample: (i) the first slice (Slice 1) was reconstructed with a circular ROE with diameter of 189 pixels and a coincident ROI of diameter 110 pixels, both centered in the image; (ii) the second slice (Slice 2) has an ROE diameter of 230 pixels and an ROI diameter of 195 pixels, off-centered from the image centre for ramp filter application onto the image, but an ROI diameter of only 160 pixels,

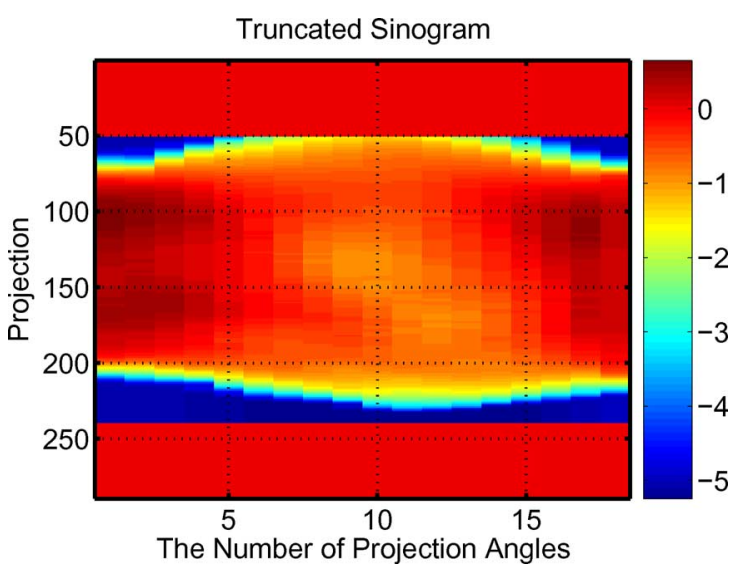

Fig. 5. Illustration of the truncated projections with $79 \%$ of full data at the center area of interest.

also off-centered from the image centre for wavelet-based reconstruction; (iii) the third slice (Slice 3) with an ROE diameter of 209 pixels and an ROI diameter of 150 pixels, off-centered from the image centre; (iv) the fourth slice (Slice 4) with an ROE diameter of 196 pixels and an ROI diameter of 140 pixels, off-centered from the image centre. Each of dataset has a pixel interval of $0.5 \mathrm{~mm}$.

\section{A. Slice One}

First consider the sinogram at Slice 1 with zero padding for nonlocal data. The reconstructed images at an off-center area with a radius of 95 pixels using the current local reconstruction algorithms. A $250 \times 250$ pixel image of the polystyrene clown's head target is recovered from scaling coefficients using local data, and the BioSpline 2.2 biorthogonal basis is used. This wavelet basis is applied to all the slices for wavelet-based reconstructions. A full wavelet reconstruction requires computing the inverse wavelet transform from four images reconstructed using separable scaling and wavelet functions. However, it is found experimentally that reconstructions using only the scaling function provides a good approximation in the region of interest—such results are presented below for illustrative purposes.

The sinogram at Slice 1 with zero padding for nonlocal data is shown in Fig. 5. The reconstructed images at an off-center 


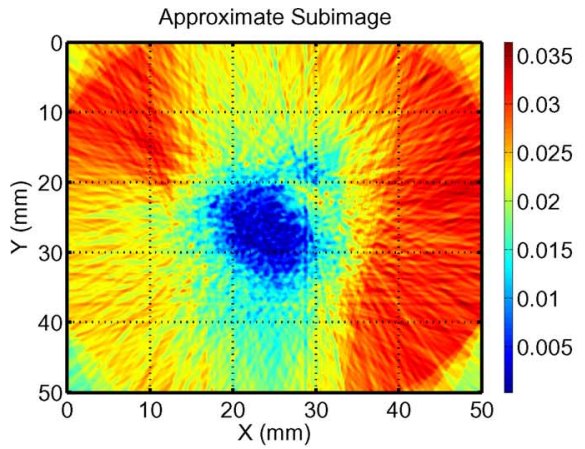

(a)

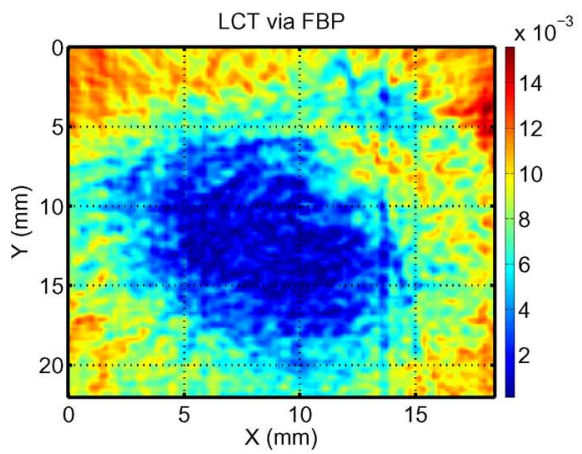

(d)

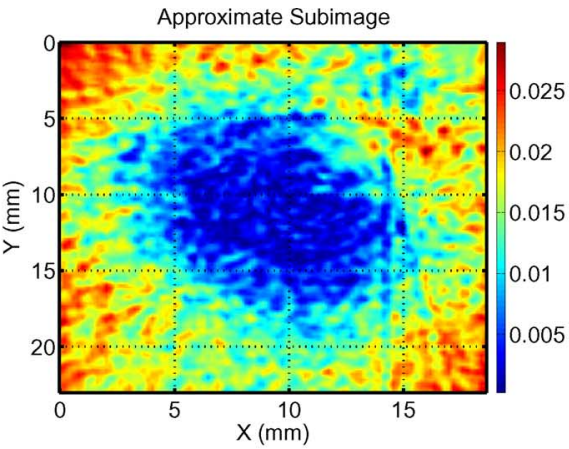

(b)
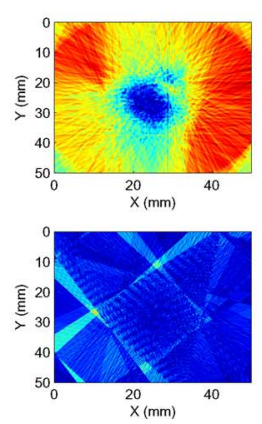

(e)

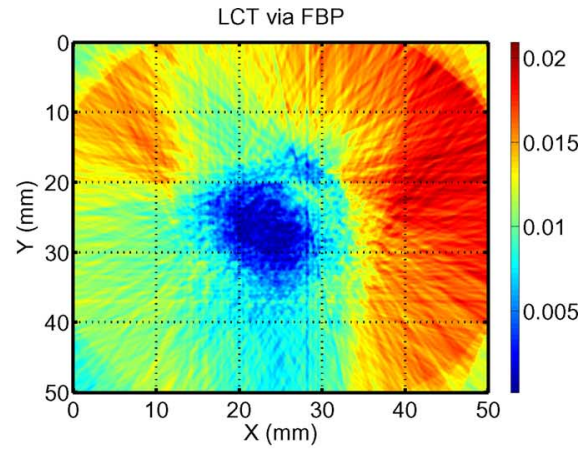

(c)

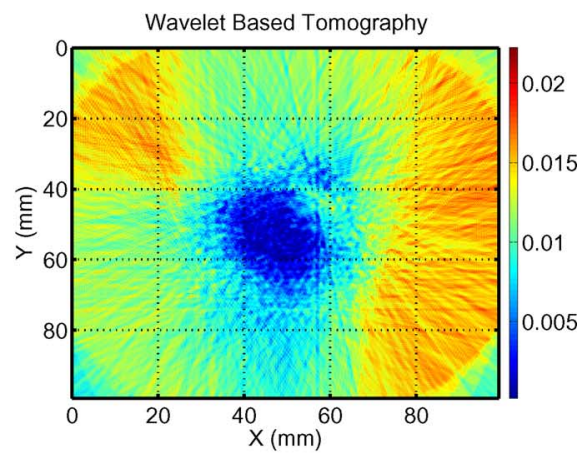

(f)

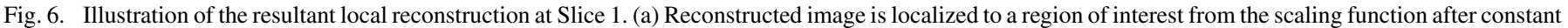

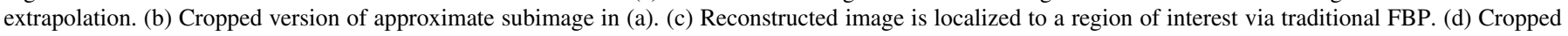

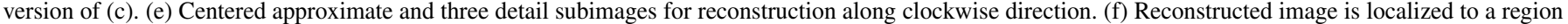
of interest from the 2-D inverse wavelet transform with interpolation.

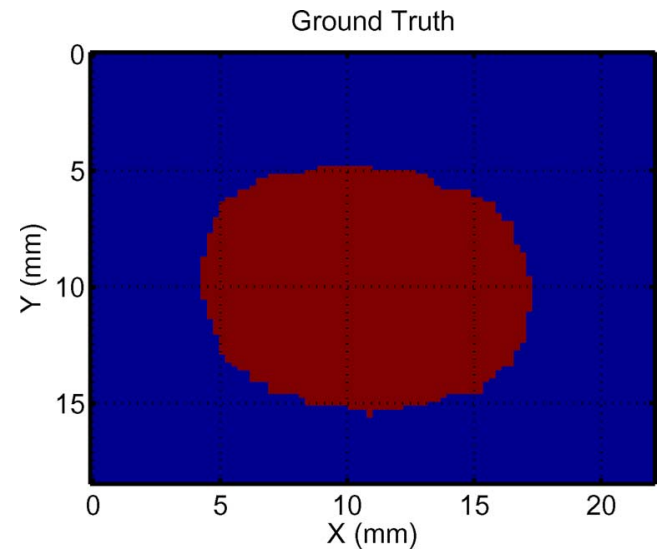

Fig. 7. Illustration of the ground truth image.

area with a radius of 95 pixels using the current local reconstruction algorithms. A $250 \times 250$ pixel image of the polystyrene clown's head target is recovered from scaling coefficients using local data, shown in Fig. 6(a), and Fig. 6(b) shows the truncated version of Fig. 6(a) showing only the region of interest (ROI). Fig. 6(c) shows that the local image with same size is recovered via traditional filtered back projection while Fig. 6(d) is the truncated version of Fig. 6(c) showing only the ROI. Wavelet and scaling coefficients after back projection are shown in Fig. 6(e). Two-dimensional inverse wavelet transforms are conducted on the four reconstructed subimages to obtain the image with full package of wavelet-based image reconstruction, shown in Fig. 6(f). It is found that in Fig. 6, the wavelet approximate subimage of (a) shows higher contrast in the reconstructed intensity than the full inverse wavelet transform reconstruction in (f), which can be illustrated via the associated color bars. This is because that the scaling function using the BioSpline 2.2 wavelet basis demonstrates an ability in filtering out high-frequency system noise from terahertz QCL imaging system, and effectively thresholds faint scattering and absorption artifacts around bright image features. Based on this observation, in the current algorithm related to wavelet-based reconstruction, we only consider the approximate image reconstruction to evaluate the algorithm validation. As we mainly deliver local information for the back projection, the wavelet and scaling ramp filters show essentially same support. But we have to recognize that having a scaling-only reconstruction means we theoretically lose some accuracy in locating any edges.

The segment of the reconstructed image via wavelet scaling sequence, Fig. 8(c), shows smoother contours with reduced artifacts than the segmented image via the FBP algorithm, Fig. 8(e). In addition, the scaling function leads to an LCT with small difference in segment from the ground truth, Fig. 8(d), compared to the difference in segment, Fig. 8(f), between the traditional local CT and the truth data, though a slightly large difference in segmentation from the truth data compared with the difference, Fig. 8(b), between traditional reconstructed segment in ROI using global data, Fig. 8(a), and the ground truth. The relative error ratio using different algorithms are shown in Table I. 


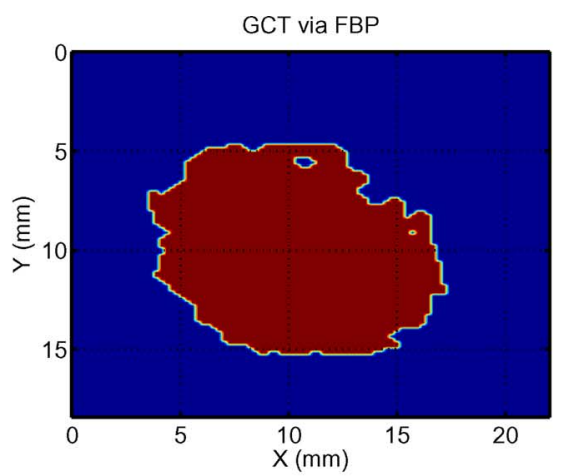

(a)

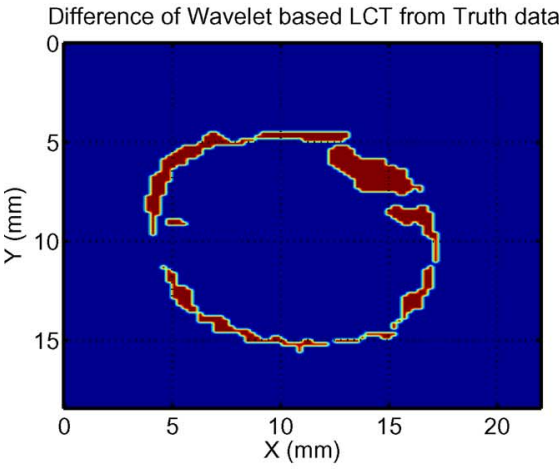

(d)

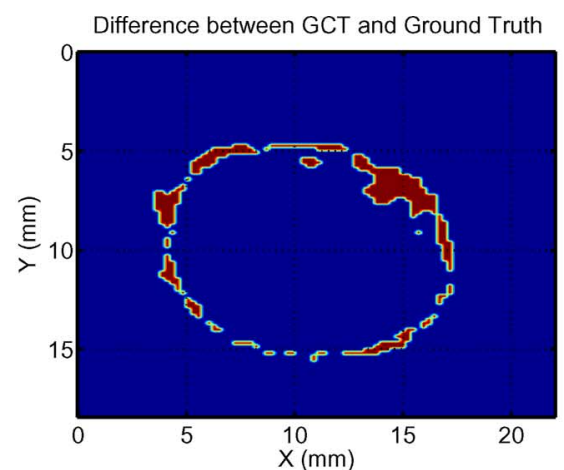

(b)

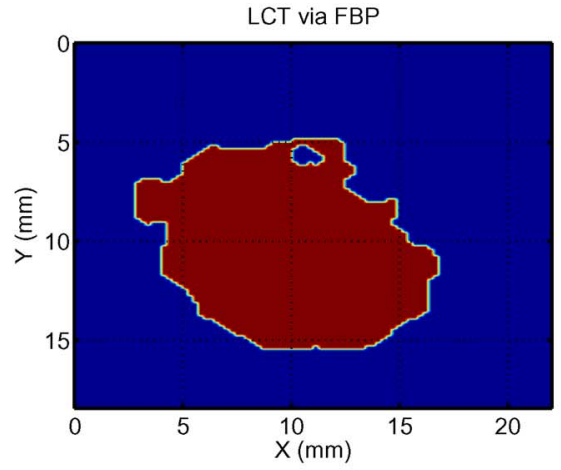

(e)

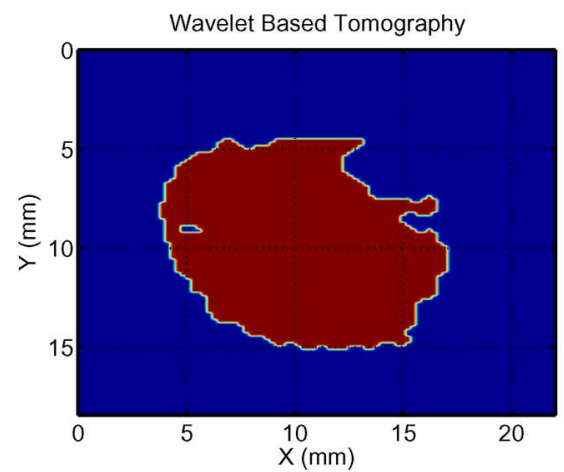

(c)

Difference between FBP based LCT and Ground Truth

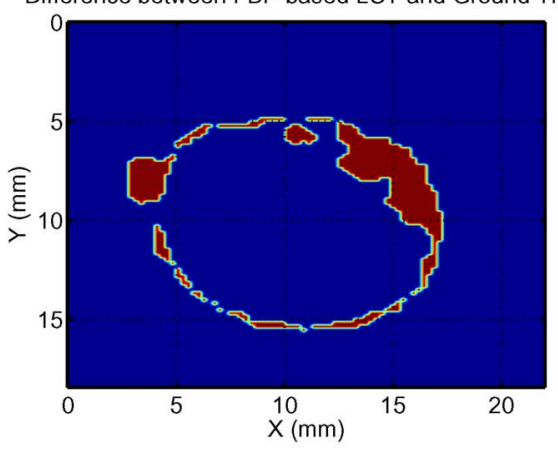

(f)

Fig. 8. Illustration of the resultant segments and the difference from the ground truth at Slice 1. (a) The segment in the ROI using traditional FBP and global data-We call it global computed tomography (GCT). (b) The difference between the segment of (a) and the ground truth. (c) The segment in the ROI using scaling function and local data. (d) The difference between the segment of (c) and the ground truth. (e) The segment in the ROI using traditional FBP and local data. (f) The difference between the segment of (e) and the ground truth.

TABLE I

Size OF Both the ROE AND the ROI AT Four TARGET HeIGHTS AND VIA THE Two DifFerent ReCONSTRUCTED AlgorithMS, IN UNITS OF PIXEL COUNT

\begin{tabular}{cccccc}
\hline Algorithms & Radius & L1 & L2 & L3 & L4 \\
\hline \hline approximate LCT & ROE & 95 & 115 & 105 & 99 \\
\hline \hline RCT via FBP & ROI & 55 & 80 & 75 & 70 \\
\hline & ROE & 95 & 115 & 105 & 99 \\
\hline & ROI & 55 & 99 & 75 & 70 \\
\hline
\end{tabular}

Generally, at this slice, the segments show good reconstruction performance whether from the traditional FBP algorithms using global and local data or from the scaling function for LCT, owing to the uniformity in the target sample and the shortest path length to the hole position.

\section{B. Slice Two}

Fig. 9(a)-(e) shows reconstructed images at an off-center area with a radius of 115 pixels using wavelet-based local reconstructions and traditional FBP algorithms. It is evident to see that to achieve the same size of the hole feature, the radius of the ROE for local objection reconstruction via FBP is larger than wavelet-based reconstruction, both of which result in similar reconstruction error to the corresponding global reconstruction (see Fig. 10). Additionally, applying FBP-based LCT to this slice leads to significant ringing problems, but the wavelet ramp filter performs much better in this regard.

Fig. 10(a)-(f) shows various reconstruction segments in the ROI and their differences from the ground truth. It is found that localized reconstructions from both the scaling function and the traditional FBP result in reduced reconstruction errors, compared to a global FBP algorithm. However, we have to recognize that the greater scattering from the irregular shape of the target sample at this height yields much more gaps after thresholding; this in turn leads to greater difficulty in the postprocessing to bridge the gaps and obtain relatively smooth segments.

\section{Slice Three}

Fig. 11(a)-(e) shows a series of reconstructed images in an off-centered region of Slice 3 with a radius of 105 pixels and $72 \%$ of full projection data. For this slice, the scattering tends to be weakened due to stronger absorption caused by the longer distance between the hole position and the QCL. As a result, compared to the image reconstruction at Slice 2, Slice 3 shows improved reconstructed accuracy as determined by the difference between the LCT and ground truth; these are illustrated in Fig. 12. In comparison with Slice 2's segmentation performance, the segments from Slice 3 have smoother boundaries and also reduced misclassification and artifacts due to the larger absorption counteracting scattering effects. Meanwhile, the local reconstruction via scaling function shows improved LCT segments in both their smoothness and classification accuracy.

\section{Slice Four}

Fig. 13(a)-(e) shows, at Slice 4, the reconstructed images at off-center areas and with a radius of 197 pixels and $74 \%$ of full projection data. 


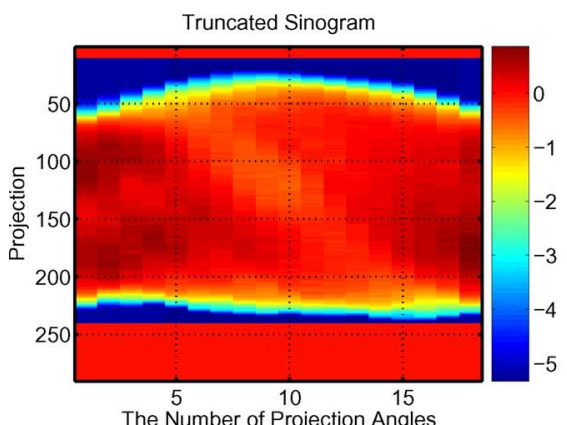

(a)

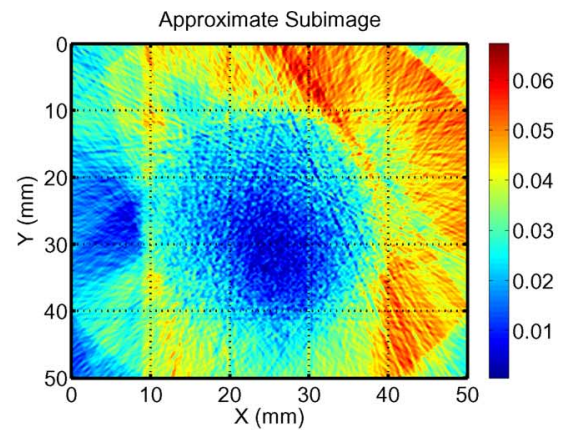

(b)

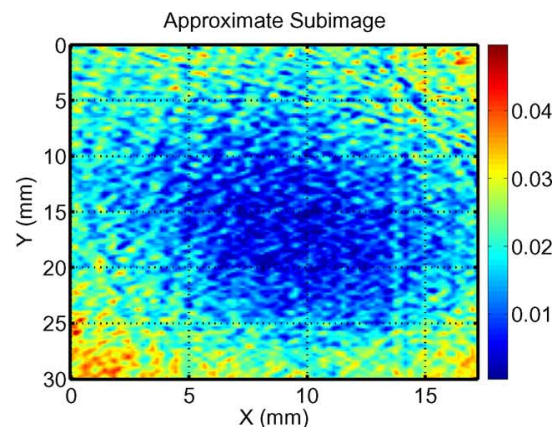

(c)

LCT via FBP

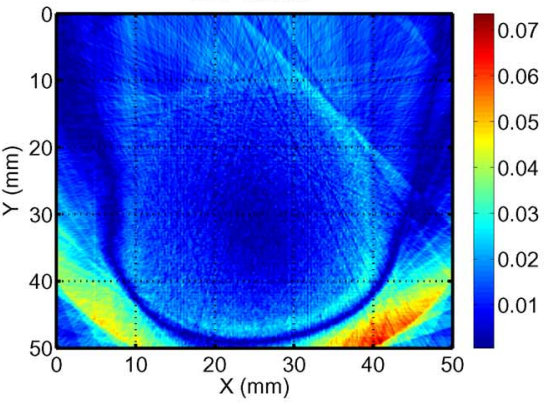

$(\mathrm{d})$

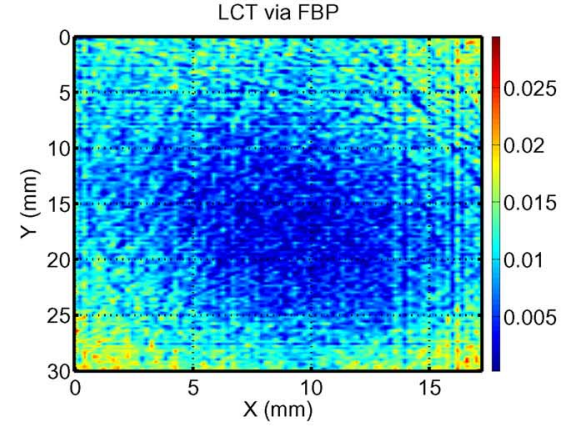

(e)

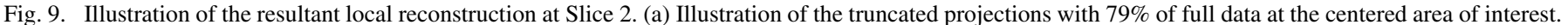

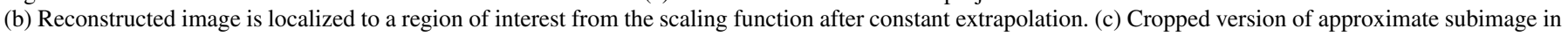
(b). (d) Reconstructed image is localized to a region of interest via traditional FBP. (e) Cropped version of (d).

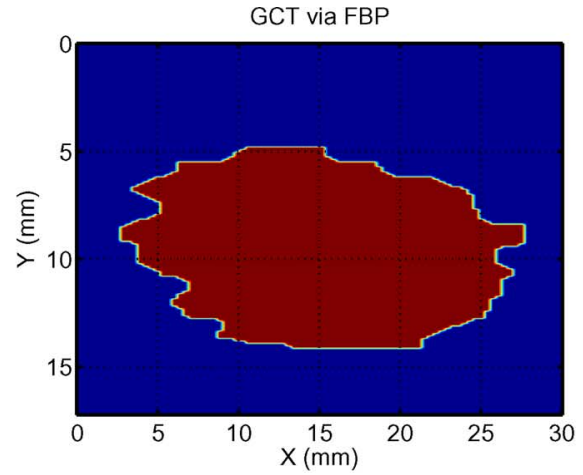

(a)

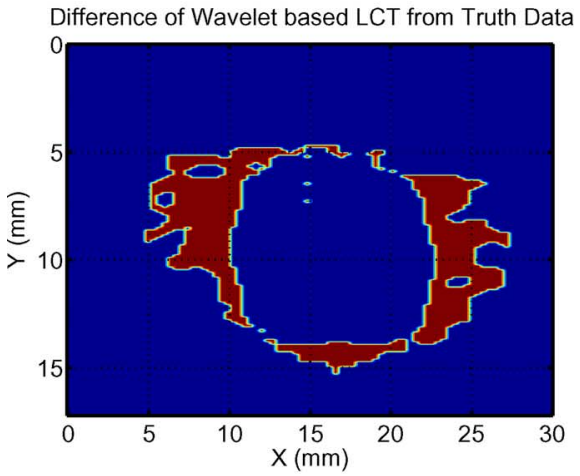

(d)

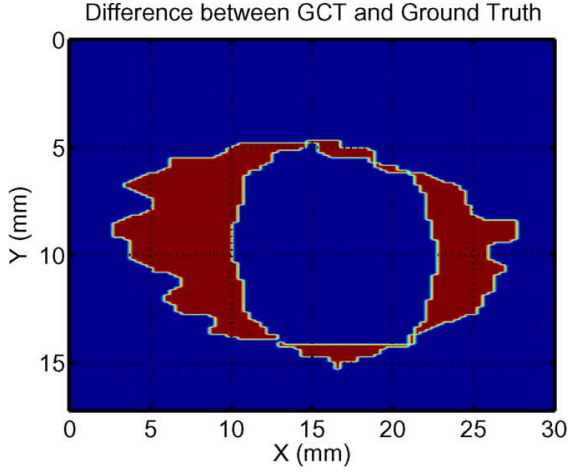

(b)

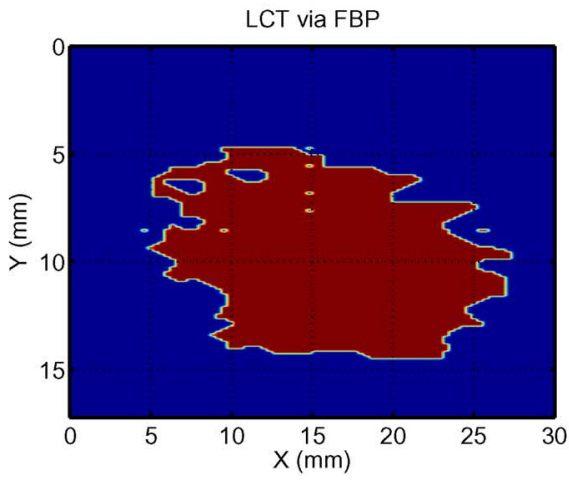

(e)

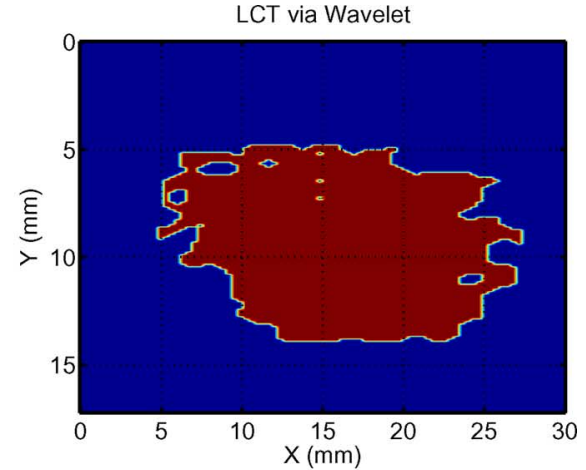

(c)

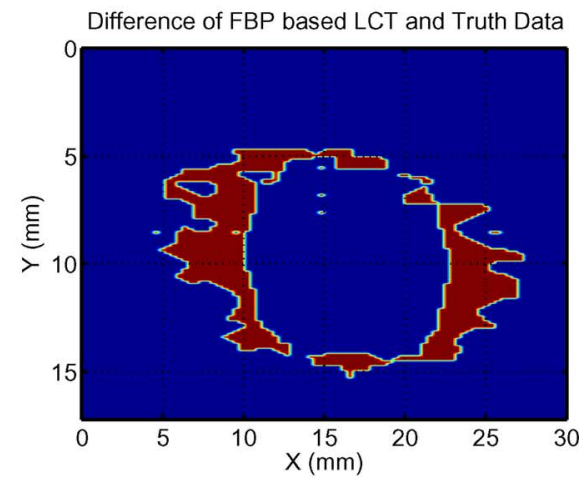

(f)

Fig. 10. Illustration of the resultant segments and the difference at Slice 2. (a) The segment in the ROI using traditional FBP and global data (GCT). (b) The difference between the segment of (a) and the ground truth. (c) The segment in the ROI using scaling function and local data. (d) The difference between the segment of (c) and the ground truth. (e) The segment in the ROI using traditional FBP and local data. (f) The difference between the segment of (e) and the ground truth. 


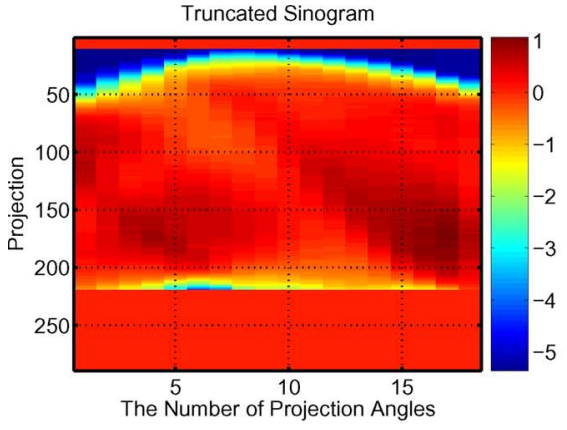

(a)

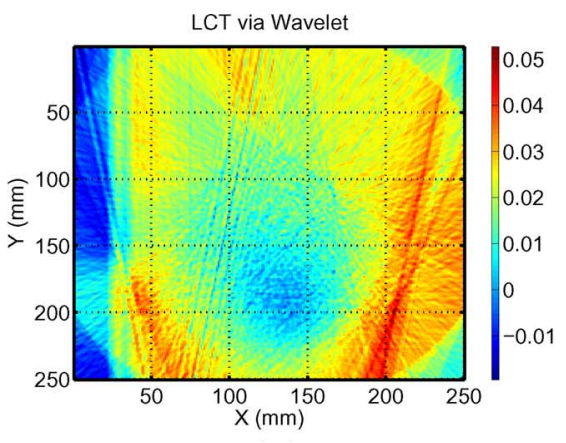

(b)

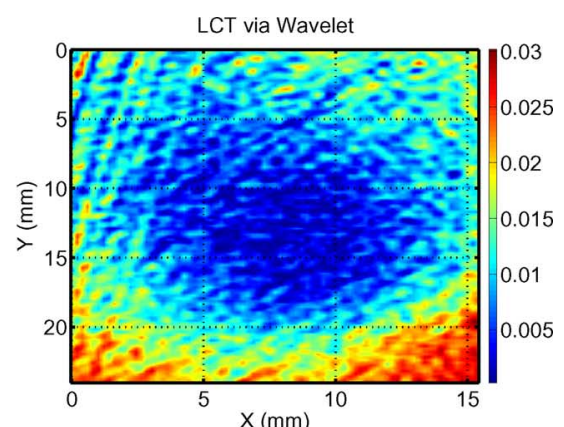

(c)

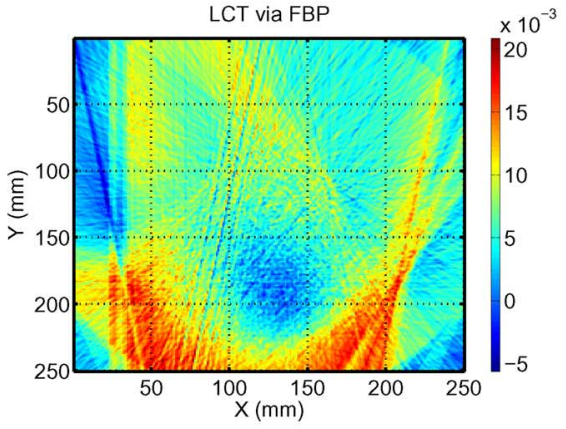

$(\mathrm{d})$

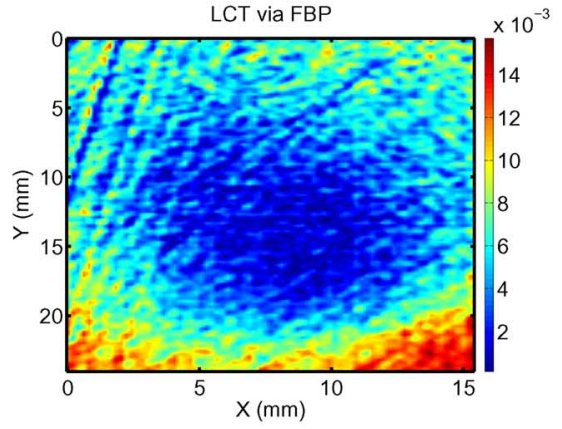

$(\mathrm{e})$

Fig. 11. Illustration of the resultant local reconstruction at Slice 3. (a) Illustration of the truncated projections with $72 \%$ of full data at the center area of interest. (b) Reconstructed image is localized to a region of interest from the scaling function after constant extrapolation. (c) Cropped version of approximate subimage of (b). (d) Reconstructed image is localized to a region of interest via traditional FBP. (e) Truncated version of (d).

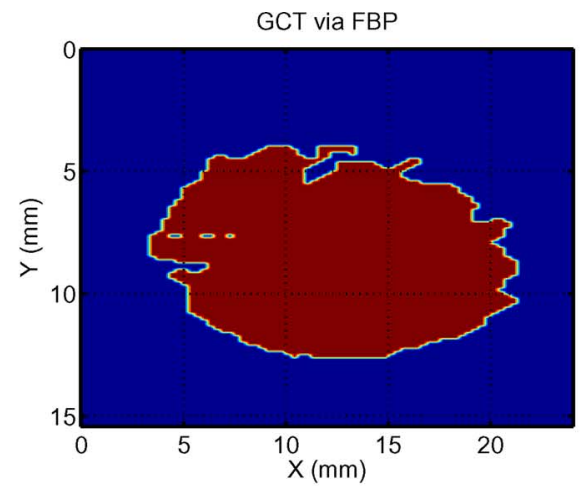

(a)

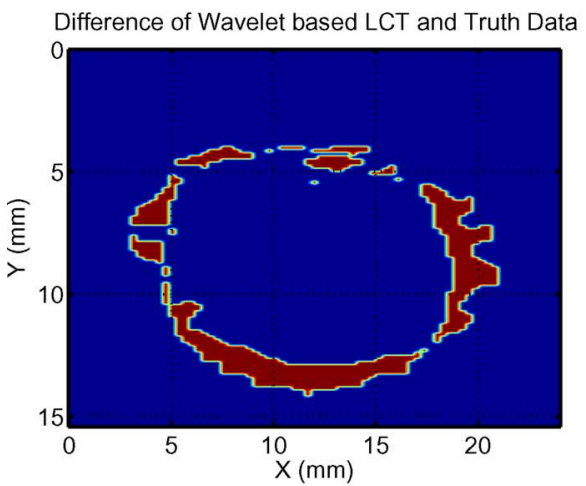

(d)

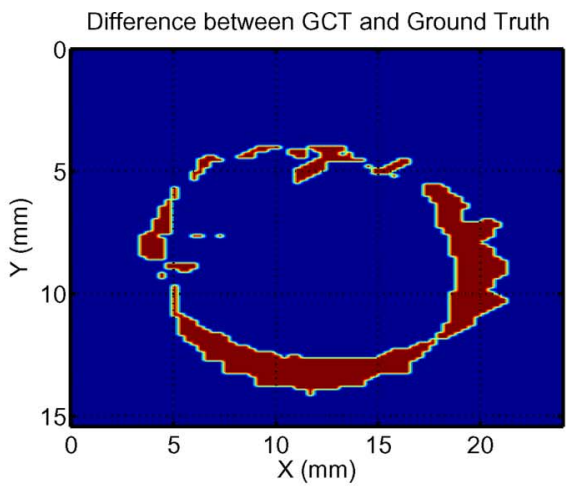

(b)

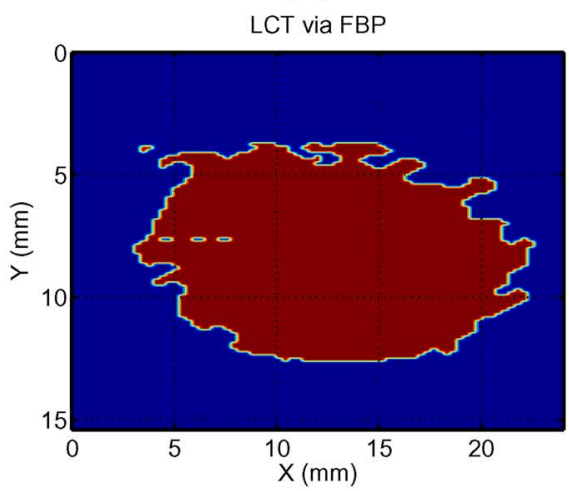

(e)

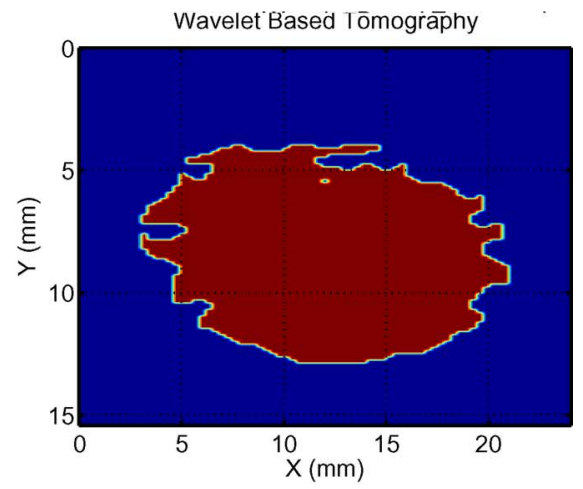

(c)

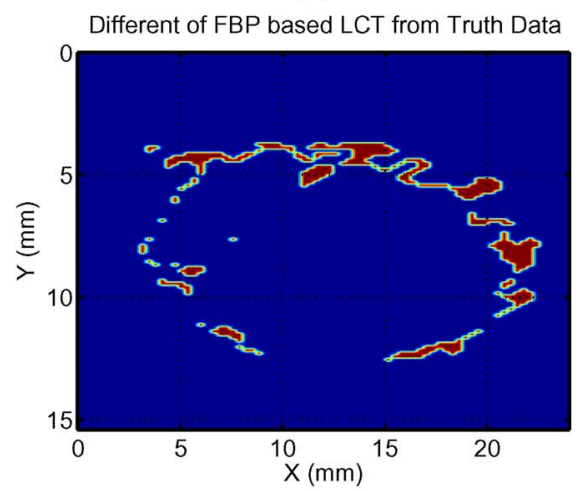

(f)

Fig. 12. Illustration of the resultant segments and the difference at Slice 3. (a) The segment in the ROI using traditional FBP and global data. (b) The difference between the segment of (a) and the ground truth. (c) The segment in the ROI using scaling function and local data. (d) The difference between the segment of (c) and the ground truth. (e) The segment in the ROI using traditional FBP and local data. (f) The difference between the segment of (e) and the ground truth.

For this slice, the exterior boundary contour of the target tends to be much smoother than that for Slices 2 and 3. Scaling func- tion reconstruction again shows the better smoothing boundary and reduced misclassification, with stronger intensity contrast in 


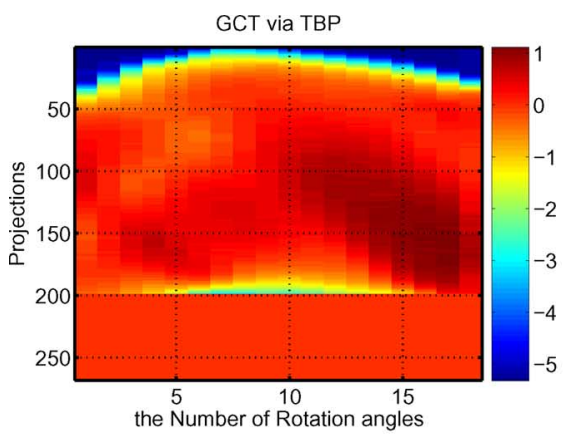

(a)

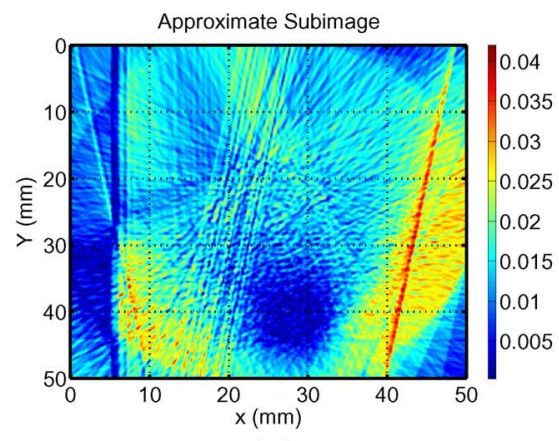

(b)

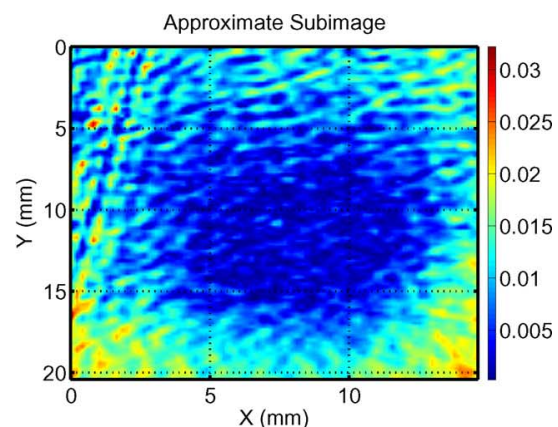

(c)

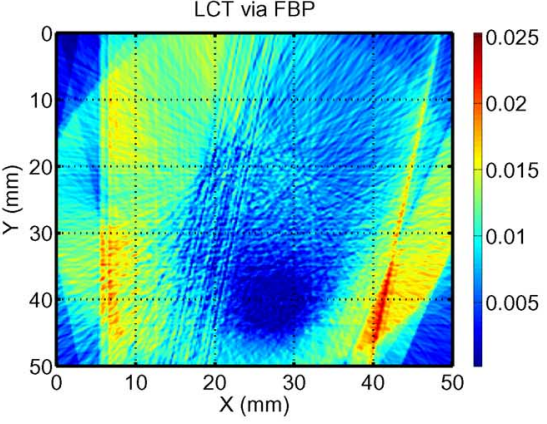

$(\mathrm{d})$

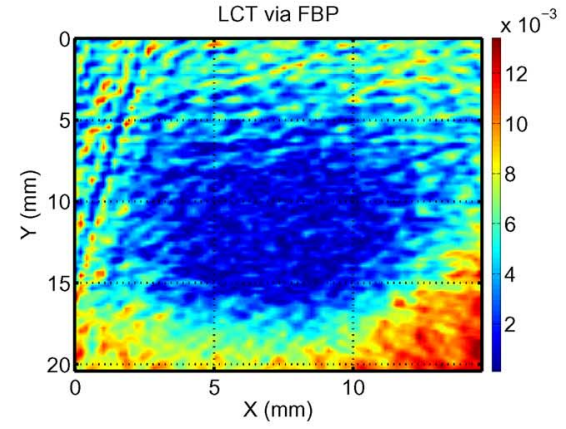

(e)

Fig. 13. Illustration of the resultant local reconstruction at Slice 4. (a) Illustration of the truncated projections with $74 \%$ of full data at the center area of interest. (b) Reconstructed image is localized to a region of interest from the scaling function after constant extrapolation. (c) Truncated version of approximate subimage of (b). (d) Reconstructed image is localized to a region of interest via traditional FBP. (e) Truncated version of (d).

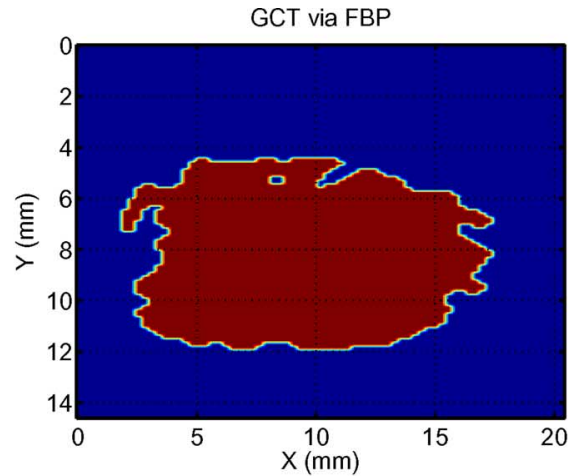

(a)

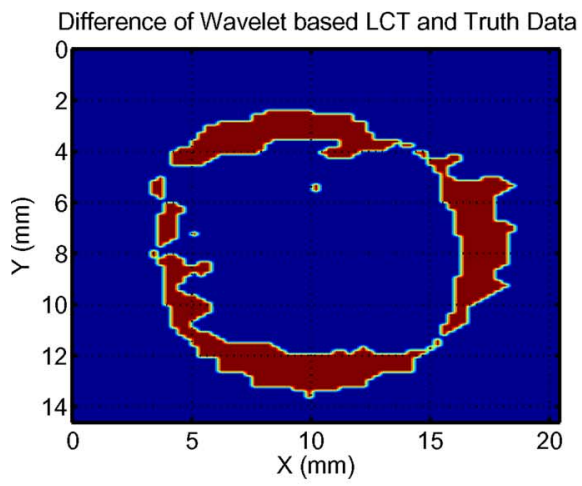

(d)

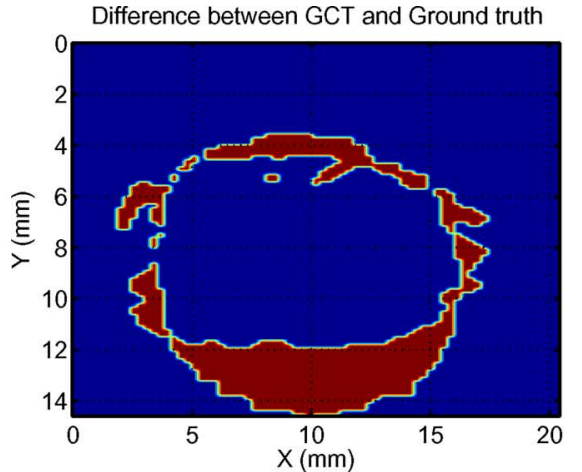

(b)

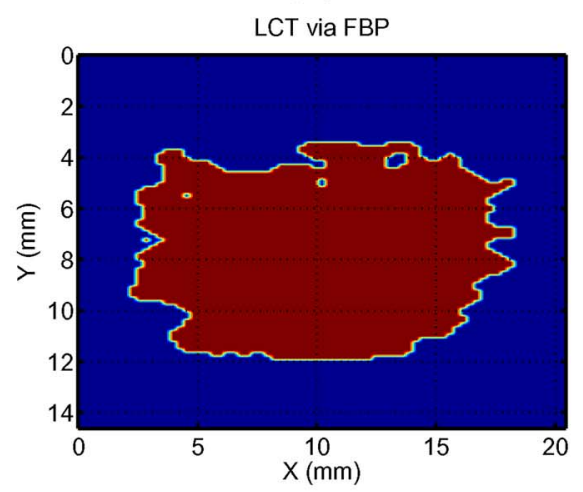

(e)

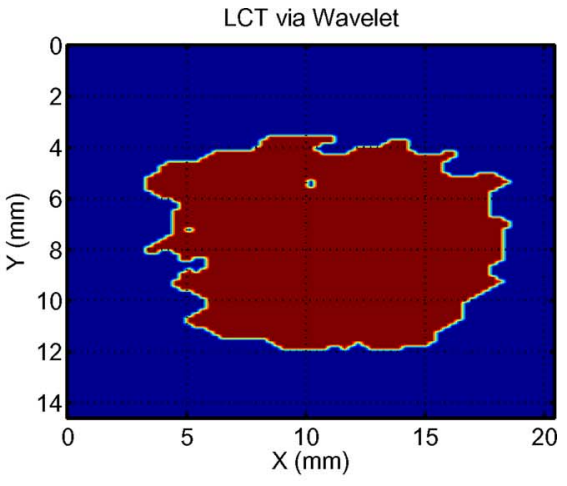

(c)

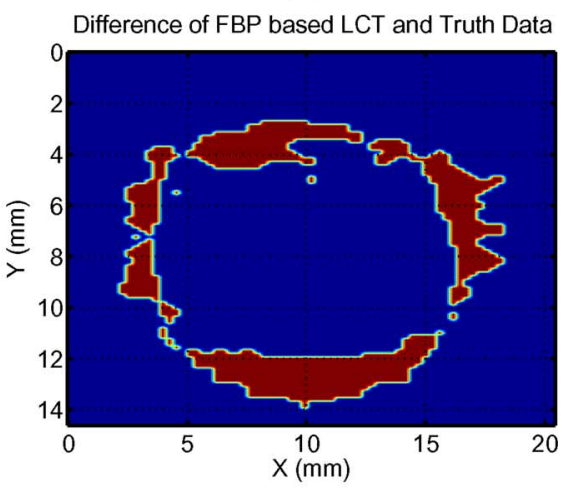

(f)

Fig. 14. Illustration of the resultant segments and the difference at Slice 4. (a) The segment in the ROI using traditional FBP and global data. (b) The difference between the segment in (a) and the truth data. (c) The segment in the ROI using scaling function and local data. (d) The difference between the segment in (c) and the truth data. (e) The segment in the ROI using traditional FBP and local data. (f) The difference between the segment in (e) and the ground truth.

the reconstructed image than the traditional FBP. The local reconstructed segments in Fig. 14(a)-(f) show much stronger ab- sorption than scattering since the longer distance from the laser area to the hole position. 
TABLE II

The CEnTroid COORDinates OF THE EXTRACTED Hole FOR EACH HeIGHT, With UNITS OF PIXEL

\begin{tabular}{cccccc}
\hline Algorithms & Coordinates & L1 & L2 & L3 & L4 \\
\hline \hline GCT via FBP & $x$ & 134 & 157 & 185 & 207 \\
\hline \hline approximate LCT & $y$ & 118 & 124 & 130 & 136 \\
\hline \hline LCT via FBP & $x$ & 135 & 156 & 184 & 198 \\
\hline & $y$ & 116 & 126 & 132 & 138 \\
\hline & $x$ & 136 & 154 & 191 & 202 \\
\hline & $y$ & 121 & 130 & 134 & 141 \\
\hline
\end{tabular}

TABLE III

THE ERROR RATIO From EACH OF THE Four HeIGHTS AND EACH OF THE THREE RECONSTRUCTED METHODS

\begin{tabular}{ccccc}
\hline Applied algorithms & L1 & L2 & L3 & L4 \\
\hline GCT via FBP & 0.1380 & 0.7112 & 0.3042 & -0.3761 \\
\hline approx. LCT & 0.1824 & 0.5306 & 0.2664 & -0.3532 \\
\hline LCT via FBP & 0.2136 & 0.5350 & 0.3824 & -0.3552 \\
\hline
\end{tabular}

\section{E. Segment Evaluation}

In order to evaluate the segmentation performance of our experiment, we exploit the fact that the internal structure, the hole, is linear with the same diameter from the top to end. This implies that the segmented hole should have the same dimensions in all slices, with only its centre displaced at various heights. To obtain this measurement, we compute the centroid of the extracted hole for each height, L1-L5, and the resultant $x$ and $y$ locations are recorded in Table II. The slopes computed using centroids from any of two successive slices are recorded in Table III below, along with the results obtained from our three reconstruction algorithms: traditional global reconstruction, scaling function used for LCT and traditional local reconstruction via FBP. For all the Tables listed below, L1-L4 mean Slices 1-4.

Table I records the radii of the ROE and ROI at different heights via applying scaling function and FBP algorithms for reconstruction. The values of ROE and ROI are selected iteratively till the reconstructed hole segment with minimum error in size compared to the "true" target. It is found that, at the height of Slice 1, the radii of the ROE and ROI are the smallest among all the measured heights, owing to the smaller optical distortion, but it also required the greatest value of (ROE-ROI). At the height of Slice 2, the biggest radii of the ROE and ROI are applied to LCT, though, the scaling function for the reconstruction needs a smaller radius of ROI than for FBP-based reconstruction. In other words, it is possible for wavelets to acquire the same quality in LCT reconstruction using a smaller exposure area. With the reduced optical distortion and improved reconstruction accuracy at the heights of Slices 3 and 4, there are reduced radii of the ROE and ROI to be employed for the local reconstruction of the similar size of hole cross-section. In summary, less scattering means requiring smaller ares of ROE to recover the same local ROI image, and vice versa.

Table II is the centroid coordinates of the extracted hole crosssection at each height, which are used to calculate slopes at different target heights.

The current slope calculated is a line segment that connects two centroids from any two reconstructed segmented images. For convenience, we call it the reconstructed slope. The error in the slope is calculated via the comparison of the value between the reconstructed slopes and the slope of two "true" centroids of the hole planar projections at two different measurement heights.

In the current local reconstruction, only the hole area is the ROI. According to a straight linear structure of tubular hole, the true projections of the hole at various heights onto $x y$ coordinate plane has the same $x$ and $y$ coordinates, the result of which is an overlapping with the origin, with slope of 0 . For the reconstructed hole segments at different heights, due to only local data involved for reconstruction, this causes difficulty in accurate recovery of an image. It turns out to be the changed positions of reconstructed centroids at the different hole segments, therefore producing altered reconstructed slopes. Subtracting the true slope of 0 from the reconstructed slope, the error in slope can be viewed as the slope of the line that passes through the centroids of any two reconstructed segments. The average slope is calculated via summing up the slopes for any two different reconstructed segments, which then is divided by the number of collected slopes. The average error in slope across all the reconstructed segments is calculated.

The average slope obtained from scaling function-only local reconstructions is 0.3355 , with reduced average error of 0.0112 compared to FBP-based local reconstruction. The slope error of the latter is 0.3467 . Since the amount of the image data used for the local reconstruction is not complete, reconstruction performance should be inferior to FBP algorithm using global data. The slope calculated using FBP algorithm on global data yields an average value of 0.2450 , which obviously means full reconstruction suffers less distortion than the remaining two local methods.

Compared with the ground truth of a slope of 0 , the global reconstruction causes the smallest error in slope among the current reconstruction algorithms. Among the local data algorithms, local reconstruction using scaling function achieves better recovery of the target region than direct FBP on the same local data.

The physical properties are indicated in Table III, via recording the calculated error ratio with respect to the reconstructed segment images on various measurement heights for different reconstruction schemes. Scattering occurs primarily with forward propagation, which involves absorption. Since the only measurable quantity is the intensity of light in the $\mathrm{CW}$ THz QCL imaging, and, due to multiple scattering, strong pathlength dispersion occurs, which results in a loss of localization and resolution. The scattering affects the image reconstruction, and it turns out that many background pixels are misclassified as hole pixels. The result is that the reconstructed target segment has the size greater than ground truth. Whereas, the absorption effect results in weakened intensity around the reconstructed boundaries of an object, the result of which is the size or the amount of pixels of a reconstructed object less than ground truth. The combined performance of absorption and scattering is such that when scattering effect is obvious compared to absorption, the resultant segment tends to have more reconstructed pixels than ground truth; when absorption effect is over scattering, the combined performs regarding the reconstructed image are the amount of reconstructed pixels less 
than ground truth. Since the current tomographic reconstruction is scattering dependent, generally, the difference between the number of reconstructed pixels and ground truth is larger than zero, in addition to the layer, where there is obvious absorption effect.

The reconstructed error ratio is represented in Table III. It is simply defined as the number of misclassified pixels/total number of pixels. According to Table III, the reconstructed error ratio is the smallest at Slice 1 and the largest at Slice 2, due to the difference in scattering caused by the variety in shape of the target sample at the different heights. The error ratio in Slice 3 is reduced compared to Slice 2 since absorption counteracts the effect of the scattering; the error ratio in Slice 4 is negative because the effect of optical absorption dominates optical scattering.

Please note that the error ratio we mean is the rate regarding the difference of the amount of the pixel between a reconstructed image and ground truth versus ground truth. Scattering occurs primarily with forward propagation, which involves absorption. Scattering accounts for significant deviations of $\mathrm{THz}$ radiation, which turns out to be a loss of localization and resolution. As a result, the amount of pixels regarding the resultant reconstruction in ROI, exceeds the amount from ground truth. This is the reason that the error we calculate is positive for Slice 1, Slice 2, and Slice 3. Whereas, at the Slice 4, absorptions of the specimen suppress optical scattering effect. The performance of the reconstructed slice image is the weakened intensity along the target contour. The result is shape shrinkage and loss of details in a recovered image. The resultant segment performance is the smaller amount of image pixels in ROI than the ground truth. Therefore, the resultant error ratio is negative.

\section{CONCLUSION}

We extend a wavelet-based algorithm to reconstruct wavelet and scaling coefficients of a target object image from its Radon transform, obtained experimentally using terahertz signals generated by a QCL. The algorithm is based on the observation that for some wavelet bases, with sufficient zero moments, the scaling and wavelet functions have essentially the same support after ramp filtering. Using experimental data obtained on a 3-D target with internal structure, we have shown that wavelet-based reconstructions offer robust reconstruction performance in the local reconstructed shape of the target. Segmentation is used in postprocessing to make measurements on the target's internal structure, and the results are analyzed to determine the effects of the optical distortion produced by a variable exterior boundary at the different cross-sections. A reconstruction utilizing only the scaling function shows a slight structure distortion in the reconstruction of a 3-D structure compared to global CT, due to the use of less data, but is superior to a traditional FBP algorithm using local data.

\section{ACKNOWLEDGMENT}

The authors thank J. Freeman and V. Apostolopoulos, for their contributions to the hardware set up shown in Fig. 1(b) and R. Rungsawang for her assistance with the terahertz quantum cascade laser imaging setup.

\section{REFERENCES}

[1] J. Faist, F. Capasso, D. L. Sivco, C. Sirtori, A. L. Hutchinson, and A. Y. Cho, "Quantum cascade laser," Science, vol. 5158, pp. 553-556, 1994.

[2] M. Belkin, F. Capasso, A. Belyanin, D. Sivco, A. Cho, D. Oakley, C. Vineis, and G. Turner, "Terahertz quantum-cascade-laser source based on intracavity difference-frequency generation," Nature Photonics, vol. 1, pp. 288-292, 2007.

[3] C. Gmachl, F. Capasso, D. L. Sivco, and A. Y. Cho, "Recent progress in quantum cascade lasers and applications," Report on Progress in Physics, vol. 64, pp. 1533-1601, 2001.

[4] M. Tonouchi, "Cutting-edge terahertz technology," Nature Photonics, vol. 1, no. 2, pp. 97-105, 2007.

[5] R. Köhler, R. C. Iotti, A. Tredicucci, and F. Rossi, "Design and simulation of terahertz quantum cascade lasers," App. Phys. Lett., vol. 79, pp. 3920-3922, 2001.

[6] A. Tredicucci, R. Köhler, L. Mahler, H. E. Beere, E. H. Linfield, and D. A. Ritchie, "Quantum cascade laser," Semiconductor Sci. Technol., vol. 20, no. 7, pp. S222-S227, 2005.

[7] L. Ozyuzer, A. E. Koshelev, C. Kurter, N. Gopalsami, Q. Li, M. Tachiki, K. Kadowaki, T. Yamamoto, H. Minami, H. Yamaguchi, T. Tachiki, K. E. Gray, W.-K. Kwok, and U. Welp, "Emission of coherent THz radiation from superconductors," Science, vol. 318, no. 5854, pp. 1291-1293, 2007.

[8] D. Abbott and X.-C. Zhang, "T-ray imaging, sensing, and retection," Proc. IEEE, vol. 95, pp. 1509-1513, Aug. 2007.

[9] S. M. Kim, F. Hatami, J. S. Harris, A. W. Kurian, J. Ford, D. King, G. Scalari, M. Giovannini, N. Hoyler, J. Faist, and G. Harris, "Biomedical terahertz imaging with a quantum cascade laser," App. Phys. Lett., vol. 88, no. 15, Art. No. 153903, 2004.

[10] X. C. Zhang, "Three-dimensional terahertz wave imaging," Philosophical Trans. Royal Society London A, vol. 362, pp. 283-299, 2004.

[11] V. Ntziachristos, J. Ripoll, L. V. Wang, and R. Weissleder, "Looking and listening to light: The evolution of whole-body photonic imaging," Nature Biotechnol., vol. 23, no. 3, pp. 313-320, 2005.

[12] S. Barbieri, J. Alton, H. E. Beere, J. Fowler, J. Ford, E. H. Linfield, and D. A. Ritchie, "2.9 THz quantum cascade lasers operating up to $70 \mathrm{~K}$ in continuous wave," App. Phys. Lett., vol. 85, no. 10, pp. 1674-1676, 2004.

[13] C. Sirtori, C. Gmachl, F. Capasso, J. Faist, D. L. Sivco, A. L. Hutchinson, and A. Y. Cho, "Long-wavelength (lambda approximately $=8-11.5 \mu \mathrm{m}$ ) semiconductor lasers with waveguides based on surface plasmons," Opt. Lett., vol. 23, pp. 1366-1368, 1998.

[14] M. Rochat, G. Scalari, D. Hofstetter, M. Beck, J. Faist, H. E. Beere, A. G. Davies, E. H. Linfield, and D. A. Ritchie, "Continuous-wave operation of far-infrared quantum cascade lasers," Electron. Lett., vol. 38, no. 25, pp. 1675-1676, 2002.

[15] R. Köhler, A. Tredicucci, F. Beltram, H. E. Beere, E. H. Linfield, A. G. Davies, and D. A. Ritchie, "High-intensity interminiband terahertz emission from chirped superlattices," App. Phys. Lett., vol. 80, no. 11, pp. 156-159, 2002.

[16] R. Köhler, A. Tredicucci, F. Beltram, H. E. Beere, E. H. Linfield, A. G. Davies, D. A. Ritchie, R. C. Iotti, and F. Rossi, "Terahertz semiconductor heterostructure laser,' Nature, vol. 417, no. 16, pp. 156-159, 2002.

[17] B. S. Williams, H. Callebaut, S. Kumar, Q. Hu, and J. L. Reno, "3.4-THz quantum cascade laser based on longitudinal-optical-phonon scattering for depopulation," App. Phys. Lett., vol. 82, no. 7, pp. 1015-1017, 2003.

[18] K. Wang and D. M. Mittleman, "Metal wires for terahertz wave guiding," Nature, vol. 432, pp. 376-379, 2004.

[19] S. Oh, J. Kang, I. Maeng, J. Suh, Y. Huh, S. Haam, and J. Son, "Nanoparticle-enabled terahertz imaging for cancer diagnosis," Opt. Express, vol. 17, pp. 3469-3475, 2009.

[20] K. M. Hanson and G. W. Wecksung, "Bayesian approach to limitedangle reconstruction in computed tomography," J. Opt. Soc. Amer., vol. 73, no. 11, pp. 1501-1509, 1983.

[21] A. C. Kak and M. Slaney, Principles of Computerized Tomographic Imaging. New York: IEEE Press, 1988.

[22] B. Ferguson, S. Wang, D. Gray, D. Abbott, and X. Zhang, "Toward functional 3 D T -ray imaging," Phys. Med. Biol. (IOP), vol. 47, pp. 3735-3742, 2002.

[23] X.-X. Yin, B. W.-H. Ng, B. Ferguson, S. P. Mickan, and D. Abbott, "2-D wavelet segmentation in 3-D T-ray tomography," IEEE Sensors J., vol. 7, pp. 342-343, 2007.

[24] K. L. Nguyen, L. J. Michael, L. Gladden, C. H. Worrall, P. Alexander, H. E. Beere, M. Pepper, D. A. Ritchie, J. Alton, S. Barbieri, and E. H. Linfield, "Three-dimensional imaging with a terahertz quantum cascade laser," Opt. Express, vol. 14, no. 6, pp. 2123-2129, 2006.

[25] R. C. Gonzalez and R. E. Woods, Digital Image Processing. Englewood Cliffs, NJ: Prentice-Hall, 2002. 
[26] Y. Fisher, Fractal Image Encoding and Analysis: A NATO ASI Series Book. New York: Springer-Verlag, 1996.

[27] F. Rashid-Farrokhi, K. Liu, C. Berenstein, and D. Walnut, "Wavelet-based multiresolution local tomography," IEEE Trans. Image Processing, vol. 6, pp. 1412-1430, Oct. 1997.

[28] T. Olson and J. DeStrfano, "Wavelet localization of the radon transform," IEEE Trans. Signal Processing, vol. 42, pp. 2055-067, Aug. 1994.

[29] A. H. Delaney and Y. Bresler, "Multiresolution tomographic reconstruction using wavelets," IEEE Trans. Image Processing, vol. 4, pp. 799-813, Jun. 1995.

[30] M. D. Levine, Vision in Man and Machines. New York: McGrawHill, 1985.

[31] R. Haralick and L. Shapiro, "Image segmentation techniques," Computer Vision, Graphics, and Image Processing, vol. 29, pp. 100-132, 1985.

[32] D. Mukherjee, P. Pal, and J. Das, "Sodar image segmentation by fuzzy c-means," Signal Process., vol. 54, pp. 295-301, 1996.

[33] K. S. Chuang, H. L. Tzeng, S. Chen, J. Wu, and T. Chen, "Fuzzy c-means clustering with spatial information for image segmentation," Comput. Med. Imag. Graph., vol. 30, pp. 9-15, 2006.

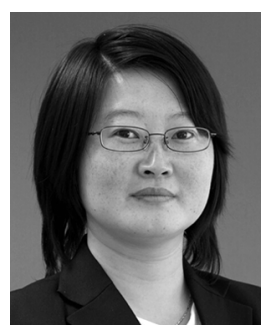

Xiaoxia (Sunny) Yin (S'07) was born in Dalian, China. She received the B.Eng. degree in industrial electronics from Dalian University, China, and the Ph.D. degree from the University of Adelaide, Adelaide, Australia, in 2009, on three-dimensional terahertz computed tomographyunder D. Abbott and B. Ng.

She was a Visiting Scholar at the University of Reading, U.K., under S. Hadjiloucas and at the University of Cambridge, U.K., under L. F. Gladden in 2008 .

Dr. Yin received a series of awards, including the Ph.D. Scholarship of the University of Adelaide (2005), the WavE Travel Fellowship from Switzerland (2006), the International Association for Pattern Recognition (IAPR) travel stipend from Hong Kong (2006), the Research Abroad Scholarship from the University of Adelaide (2006), the Roger Pysden Memorial Fellowship from Australia Business, ABL State Chamber (2006), a student scholarship from 2007 the IEEE International Symposium on Industrial Electronics, Vigo, Spain (2007), a Mutual Community Postgraduate Travel Grant from the University of Adelaide (2007), an Overseas Travel Fellowship from the Australian Research Council Nanotechnology Network (ARCNN) (2007), and the Gertrude Rohan Prize (2010). In 2009, she won a prestigious Australian Research Council (ARC) postdoctoral fellowship.

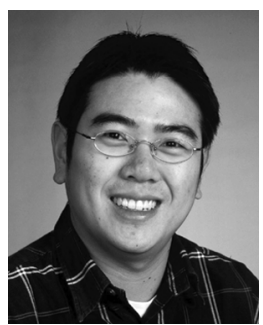

Brian Wai-Him Ng (S'97-M'02) was born in Hong Kong on May 12, 1974. He received the B.Sc. degree in mathematics and computer science, and the B.Eng. degree (Hons), and the Ph.D. degree under A. Bouzerdoum both in electrical and electronic engineering from the University of Adelaide, Adelaide, Australia, in 1996, 1997, and 2003, respectively.

Since 2002, he has been a Lecturer at the School of Electrical and Electronic Engineering, University of Adelaide. His research interests include signal processing, bio-inspired engineering, distributed sensor networks, software defined radio, as well as signal processing for T-ray imaging.

Dr. Ng was awarded the University of Adelaide Medal for the Top Graduate in Electrical and Electronic Engineering. He is currently an active member within the South Australian Chapter of the IEEE.

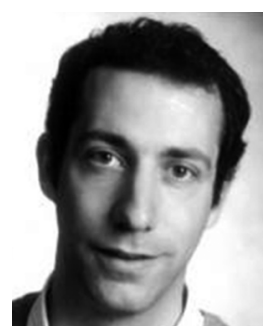

J. Axel Zeitler received the Staatsexamen from Würzburg, Germany, in 2002, the PGCert degree and the Ph.D. degree under T. Rades and K. Gordon from the University of Otago, Dunedin, New Zealand, in 2003 and 2007, respectively.

$\mathrm{He}$ is currently a Research Fellow at Gonville \& Caius College, Cambridge. He holds a Fellowship with the Department of Chemical Engineering and Biotechnology, University of Cambridge, where he previously worked as a Research Associate. Prior to that he was with the School of Pharmacy, the University of Otago, the Cavendish Laboratories, University of Cambridge, UK; and TeraView Ltd.

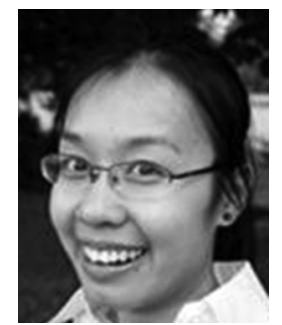

Kieu Lien Nguyen was with the Hanoi University of Medicine and won a scholarship to the University of Adelaide, Australia, graduating with a Degree in chemical engineering in 2003. She received the Ph.D. degree entitled Terahertz Spectroscopy and Imaging in Chemical Engineering in 2006.

Dr. Nguyen won Australia's Joint Chemical Engineering Committee (JCEC) Medal and the EssoMobil Outstanding Scholar Award, both for chemical engineering. In 2003, she won the Bill Gates Special Scholarship and the Honor Scholarship, sponsored by Queen Elizabeth II, for Ph.D. study at the University of Cambridge. Dr Nguyen currently works as an engineer at TWI Ltd. in the UK, specializing in applying high power lasers in the manufacturing industry.

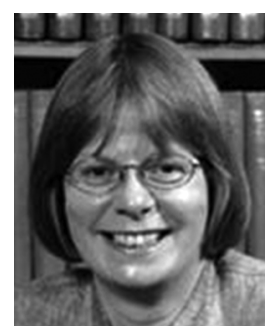

Lynn F. Gladden received the B.Sc. degree in chemical physics from the University of Bristol, Bristol, U.K., in 1982 and the Ph.D. degree in physical chemistry at the University of Cambridge, Cambridge, U.K., in 1987.

She is currently the Shell Professor of Chemical Engineering and Head of the Department of Chemical Engineering and Biotechnology, University of Cambridge. She has been made the University of Cambridge's new Pro-Vice-Chancellor for Research, in providing institutional leadership for the university. She currently heads up Cambridge's Magnetic Resonance Research Centre, and has a particular interest in applying magnetic resonance imaging techniques in the fields of heterogeneous catalysis and multiphase transport in porous media, as well as an interest in the terahertz region of the electromagnetic spectrum.

Dr. Gladden was appointed Fellow of Trinity College in 1999; Fellow of the Royal Academy of Engineering in 2003; Fellow of the Royal Society in 2004 Officer of the Order of the British Empire (OBE) in 2001; and was appointed Commander of the Order of the British Empire (CBE) in 2009.

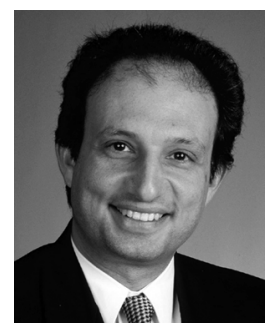

Derek Abbott (M'85-SM'99-F'05) was born on May 3, 1960, in South Kensington, London, U.K. $\mathrm{He}$ received the B.Sc. degree (Hons.) in physics from Loughborough University of Technology, U.K., in 1982 and the Ph.D. degree in electrical and electronic engineering from the University of Adelaide, Australia, in 1995, under K. Eshraghian and B. R. Davis.

From 1978 to 1986, he worked at the GEC Hirst Research Centre, London, in the areas of semiconductors and optoelectronics. On migration to Australia, he worked for Austek Microsystems, Technology Park, South Australia, in 1986. Since 1987, he has been with the University of Adelaide, where he is currently a Full Professor at the School of Electrical and Electronic Engineering. He holds over 300 publications/patents and has been an invited speaker at over 80 institutions, including Princeton, NJ; MIT, MA; Santa Fe Institute, NM; Los Alamos National Laboratories, NM; Cambridge, U.K.; and EPFL, Lausanne, Switzerland. He coauthored the book Stochastic Resonance (Cambridge University Press), and coedited the book Quantum Aspects of Life (Imperial College Press). His interests are in the area of complex systems and multidisciplinary applications of physics and engineering.

Prof. Abbott is a Fellow of the Institute of Physics (IOP), with honorary life membership. He won the GEC Bursary (1977), the Stephen Cole the Elder Prize (1998), the E.R.H. Tiekink Memorial Award (2002), SPIE Scholarship Award for Optical Engineering and Science (2003), the South Australian Tall Poppy Award for Science (2004), and the Premier's SA Great Award in Science and Technology for outstanding contributions to South Australia (2004). He has served as an Editor and/or Guest Editor for a number of journals includingthe IEEE JOURNAL OF SOLID-STATE CIRCUITS, Chaos (AIP), Smart Structures and Materials (IOP), Journal of Optics B (IOP), Microelectronics Journal (Elsevier), Fluctuation Noise Letters (World Scientific), and is currently on the Editorial Boards of the PROCEEDINGS OF THE IEEE and IEEE PHOTONICS. He has appeared on national and international television and radio and has also received scientific reportage in New Scientist, The Sciences, Scientific American, Nature, The New York Times, and Sciences et Avenir. He has served on a number of IEEE technical program committees, including the IEEE APCCS and the IEEE GaAs IC Symposium. 